\title{
Solar X-ray Monitor On Board the Chandrayaan-2 Orbiter: In-flight Performance and Science Prospects
}

\author{
N. P. S. Mithun ${ }^{1,2}$ - Santosh V. \\ Vadawale $^{1}$ (1) - Aveek Sarkar ${ }^{1}$ (D) M. \\ Shanmugam $^{1}$ - Arpit R. Patel ${ }^{1}$ (D) Biswajit \\ Mondal $^{1}$ - Bhuwan Joshi ${ }^{1}$ [D J Janardhan \\ P. ${ }^{1}$ - Hiteshkumar L. Adalja ${ }^{1}$. Shiv Kumar \\ Goyal $^{1}$ (D) Tinkal Ladiya ${ }^{1}$ - Neeraj Kumar \\ Tiwari $^{1} \cdot$ Nishant Singh $^{1} \cdot$ Sushil Kumar ${ }^{1}$. \\ Manoj K. Tiwari ${ }^{3}$ (D) M. H. Modi ${ }^{3}$ - Anil \\ Bhardwaj $^{1}$ (D) \\ (C) Springer $\bullet \bullet \bullet \bullet$
}

\begin{abstract}
The Solar X-ray Monitor (abbreviated as XSM) on board India's Chandrayaan-2 mission is designed to carry out broadband spectroscopy of the Sun from lunar orbit. It observes the Sun as a star and measures the spectrum every second in the soft X-ray band of $1-15 \mathrm{keV}$ with an energy resolution better than $180 \mathrm{eV}$ at $5.9 \mathrm{keV}$. The primary objective of the XSM is to provide the incident solar spectrum for the X-ray fluorescence spectroscopy experiment on the Chandrayaan-2 orbiter, which aims to generate elemental abundance maps of the lunar surface. However, observations with the XSM can independently be used to study the Sun as well. The Chandrayaan-2 mission was launched on 22 July 2019, and the XSM began nominal operations, in lunar orbit, from September 2019. The in-flight observations, so far, have shown that its spectral performance has been identical to that on the ground. Measurements of the effective area from ground calibration were found to require some refinement, which has been carried out using solar observations at different incident angles. It also has been shown that the XSM is sensitive enough to detect solar activity well below A-class. This makes the investigations of microflares and the quiet solar corona feasible in addition to the study of the evolution of physical parameters during intense flares. This article presents the in-flight performance and
\end{abstract}

\footnotetext{
$\varangle$ Mithun

mithun@prl.res.in

1 Physical Research Laboratory, Navrangpura, Ahmedabad, 380 009, India

2 Indian Institute of Technology Gandhinagar, Palaj, Gandhinagar, 382 355, India

3 Raja Ramanna Centre for Advanced Technology, Indore, 452 013, India
} 
calibration of the XSM instrument and discusses some specific science cases that can be addressed using observations with the XSM.

Keywords: Techniques: Spectroscopy; Sun: Corona, Flares, X-rays

\section{Introduction}

The Solar X-ray Monitor (XSM) is an X-ray spectrometer on board the orbiter of Chandrayaan-2, the second Indian mission to the Moon (Vanitha et al., 2020), and is a part of the remote X-ray fluorescence spectroscopy experiment of the mission aimed at estimating abundances on the lunar surface at a global scale. The remote X-ray fluorescence spectroscopy technique has been employed in several missions to various atmosphere-less solar system bodies to determine the elemental composition of their surfaces (Bhardwaj et al., 2007; Bhardwaj, Lisse, and Dennerl, 2014). It involves measurement of the characteristic X-ray fluorescence lines of various elements emitted due to their with the incident solar $\mathrm{X}$-rays. It is possible to obtain a quantitative estimate of the abundances of major elements using this technique if the incident X-ray spectrum and the observation geometry are known. Such experiments are typically carried out from an orbital platform, and hence the information on the observation geometry is available. On the other hand, it is known that the X-rays from the Sun are highly variable in both their intensity as well as spectral shape. Thus, it is customary to have a separate instrument on the same orbital platform that provides simultaneous measurements of the solar X-ray spectrum in order to obtain quantitative estimates of the surface elemental abundances. On the Chandrayaan-2 mission, the Chandrayaan-2 Large Area Soft X-ray Spectrometer (CLASS) (Radhakrishna et al., 2020) and the Solar X-ray Monitor (XSM) (Shanmugam et al., 2020) instruments record, respectively, the fluorescence X-ray spectrum from the lunar surface and the solar X-ray spectrum.

Remote X-ray fluorescence spectroscopy experiments have been carried out on several past missions to various solar system objects, such as the Moon (Apollo15 and 16, Smart-1, Chandrayaan-1, Chang'e-2, Kaguya), Mercury (Messenger, BeppiColombo), and asteroids (NEAR, OSIRIS-REx). All these missions carried dedicated instruments for spatially integrated and spectrally resolved solar observations: X-ray Solar Monitors on board SMART-1 (Huovelin et al., 2002), Chandrayaan-1 (Alha et al., 2009), and Chang'e-2 (Dong et al., 2019); MESSENGER-SAX (Schlemm et al., 2007); Beppicolombo-SIXS (Huovelin et al., 2010); and Solar X-ray Monitors on board NEAR-Shoemaker (Trombka et al., 2001) and OSIRIS-REx (Masterson et al., 2018). Although these instruments' primary objective was to aid the measurement of elemental abundances on the surface of the solar system objects, observations with many of them have also been used for carrying out independent solar studies (Narendranath et al., 2014a; Dennis et al., 2015).

Spectroscopic observations of the Sun in X-ray wavelengths have contributed enormously to our present understanding of the fundamental parameters of the solar corona (Del Zanna and Mason, 2018). Such studies typically make use 
of the solar X-ray instruments that fall into two classes viz. X-ray imagers providing high spatial resolution images of the Sun over a broad energy range, but without or with limited spectral information (e.g., Hinode XRT) and crystal spectrometers that provide very high-resolution spectra without or with little spatial information, but over a narrow energy band (e.g., CORONAS-F RESIK). Exceptions to these two categories include RHESSI (Lin et al., 2002) that performed imaging spectroscopy in the hard X-ray band ( $>6 \mathrm{keV}$ ) and the NuSTAR mission (Harrison et al., 2013), which was meant primarily for observations of other astrophysical sources but is capable of hard X-ray imaging spectroscopy ( $>3 \mathrm{keV}$ ) of the Sun as well. Broad-band spectral measurements with these instruments allowed the solar X-ray spectrum to be modeled over a wide range of energies to probe various aspects, including the contribution of the non-thermal processes in the corona to the X-ray emission. However, as the lower energy threshold of these instruments is higher than $1 \mathrm{keV}$, there are limitations in constraining the thermal component in the emission, particularly during low solar activity. It may also be noted that RHESSI has completed its mission duration and sensitive solar observations with NuSTAR are feasible only under quiescent solar conditions.

In the absence of instruments providing imaging and broad-band spectroscopy extending down to the energy of $1 \mathrm{keV}$, instruments that carry out even spatially integrated measurements, like those on board various planetary missions, are of importance. Such measurements over a wide energy range in soft X-rays have been carried out sporadically over the past two decades by a few dedicated experiments: Solar X-ray Spectrometer (SOXS) on board GSAT-2 (Jain et al., 2005), Solar Photometer in X-rays (SphinX) on board CORONAS-Photon mission (Gburek et al., 2013), and the recent Miniature X-ray Solar Spectrometer (MinXSS) CubeSat missions (Moore et al., 2018). Given the lack of dedicated solar instruments providing broad band spectroscopy, such measurements carried out by the Chandrayaan-2 XSM aptly complement the observations from wideband X-ray imagers, narrow-band spectrometers, and hard X-ray spectrometers for investigations of the solar corona.

The XSM on board the Chandrayaan- 2 mission provides disk integrated solar spectra in the energy range of $1-15 \mathrm{keV}$ with a spectral resolution of better than $180 \mathrm{eV}$ at $5.9 \mathrm{keV}$, which is the best available so far among similar instruments that carried out such measurements. The XSM also offers the highest time cadence for such instruments: full spectrum every second and light curves in three energy bands every $100 \mathrm{~ms}$. The unique design features of the XSM allows observations over a wide dynamic range of X-ray fluxes from the quiet Sun to $\mathrm{X}$-class flares. Presently, the XSM is the only instrument operational providing soft X-ray spectral measurements of the Sun over a broad energy range.

The Chandrayaan-2 spacecraft was launched by the Geosynchronous Satellite Launch Vehicle (GSLV) MkIII-M1, on 22 July 2019. It reached its nominal circular orbit around the Moon in early September after several orbit maneuvers and the XSM began its nominal operations in lunar orbit from 12 September 2019. Inflight observations with the XSM have been used to evaluate its performance and validate and refine the ground calibration. In this article, we present the onboard performance and calibration of the XSM and prospects of solar studies with the 
instrument. Section 2 provides an overview of the instrument, observation plan, and data analysis; the onboard performance of the instrument is discussed in Section 3 and some of the science cases that can be addressed with the XSM are presented in Section 4 followed by a summary.

\section{Chandrayaan-2 Solar X-ray Monitor: An Overview}

\subsection{Instrument and Ground Calibration}

The XSM is designed to carry out spectroscopic observations of the Sun in Xray wavelengths with stable spectral performance over a wide range of solar $\mathrm{X}$-ray intensities. As the objective is to measure the disk integrated solar X-ray spectrum, the instrument does not have any imaging elements. It uses a Silicon Drift Detector (SDD) to measure the energy of individual photons and records the X-ray spectra in the 1-15 keV energy range with a cadence of one second. The detector is covered with a detector cap or collimator having a small aperture such that the entrance area of the instrument is restricted while maintaining a large field of view (FOV) of \pm 40 degree. This large FOV maximizes the visibility of the Sun as the instrument is fix-mounted on the spacecraft and the angle between the Sun vector and the instrument boresight varies over a wide range depending on the attitude configuration of the spacecraft (Vanitha et al., 2020). The choice of materials and design of the collimator ensures that the background from all other directions and any fluorescence emission is blocked, thereby ensuring that the XSM has a very low background (Mithun et al., 2020a).

The XSM employs a closed-loop control of the temperature of the detector to ensure that the spectral resolution of $\approx 175 \mathrm{eV}$ does not vary during in-flight observations where the ambient temperatures are expected to vary significantly. The unique characteristics of the detector and the readout system also make it possible to maintain this spectral performance, without significant effect of pulse pileup, up to an incident flux of about 80,000 counts $\mathrm{s}^{-1}$ (Mithun et al., 2020a), which corresponds to the M5 class of flares as discussed later. To further extend the dynamic range, the XSM includes a filter wheel mechanism which brings a $250 \mu \mathrm{m}$ beryllium window in front of the detector when the count rate exceeds a set threshold. The Be window attenuates X-rays below $2 \mathrm{keV}$ thereby, reducing the count rate and thus enabling spectral measurements even for higher intensity flares. The filter wheel also includes an Fe-55 radioactive source covered with $\mathrm{Ti}$ foil, which serves the purpose of carrying out in-flight calibration of the spectrometer. A summary of the major specifications of the instrument is given in Table 1 and a detailed discussion of the instrument design is given in Shanmugam et al. (2020).

In order to infer the incident solar spectrum from XSM observations, the instrument's spectral response needs to be calibrated. Several dedicated ground calibration experiments were carried out to determine the gain parameters of the XSM under various observing conditions of ambient temperature and Sun angle, the spectral redistribution function of the detector, and the effective area as a function of incident angle. These measurements were used to derive an 
Table 1. Specifications of the XSM.

\begin{tabular}{ll}
\hline Parameter & Specification \\
\hline Energy Range & $1-15 \mathrm{keV}$ (up to $\approx \mathrm{M} 5$ class $)$ \\
& $2-15 \mathrm{keV}$ (above $\approx \mathrm{M} 5$ class $)$ \\
Energy Resolution & $<180 \mathrm{eV} @ 5.9 \mathrm{keV}$ \\
Time cadence & $1 \mathrm{~s}$ \\
Effective area (on-axis) & $0.135 \mathrm{~mm}^{2} @ 1 \mathrm{keV}$ \\
& $0.367 \mathrm{~mm}^{2} @ 5 \mathrm{keV}$ \\
Field of view & $\pm 40 \mathrm{degree}^{2}$ \\
Filter wheel mechanism properties & $3:$ Open, Be-filter, Cal(Fe-55) \\
$\quad$ Positions & 80,000 counts s \\
$\quad$ Be-filter movement threshold flux & M5 class $)$ \\
\hline
\end{tabular}

on-ground estimate of the response matrix. A detailed description of ground calibration aspects of the XSM is presented in Mithun et al. (2020a).

\subsection{Estimation of Count Rates for Different Classes of Solar Flares}

We utilize the response of the XSM instrument obtained from ground calibration to estimate the expected count rates during solar observations at various levels of solar activity. For this purpose, the CHIANTI atomic database (Dere et al., 1997. Del Zanna et al., 2015) was used. Synthetic solar spectra were generated considering isothermal plasma emission comprising of both continuum and lines. This was done for a range of temperatures and emission measures that span different classes of solar flares based on the correlations from Feldman et al. (1995). The upper panel of Figure 1 shows such synthetic spectra for flare classes ranging from A1 to X1 while the lower panel shows the expected spectra from observations with the XSM obtained by convolving the model spectra with the on-axis response. In the case of an X1 class flare, the dotted line shows the spectrum without the beryllium filter and the solid line shows the spectrum with the beryllium filter placed in front of the detector thereby increasing the low energy threshold to $\approx 2 \mathrm{keV}$.

Since the XSM is an instrument with a wide FOV, the most important source of background is the diffuse Cosmic X-ray Background (CXB), which will determine the sensitivity of the instrument. We estimate the expected background spectrum in the XSM using the CXB spectral model given by Türler et al. (2010). The CXB spectrum per unit solid angle obtained from the model is multiplied with the solid angle within the FOV of the XSM to obtain the incident CXB spectrum, which is shown with a black dashed line in the top panel of Figure 1. This model spectrum is convolved with the XSM response matrix and is shown with a black dashed line in the bottom panel of the same figure. The total count rate due to $\mathrm{CXB}$ is 0.1 counts $\mathrm{s}^{-1}$. It is seen from the figure that the estimated background spectrum is about two orders of magnitude below that of the A1 class spectrum at lower energies. Hence, it is expected that the XSM can provide spectral measurements, even when solar activity is below the A1 class. 

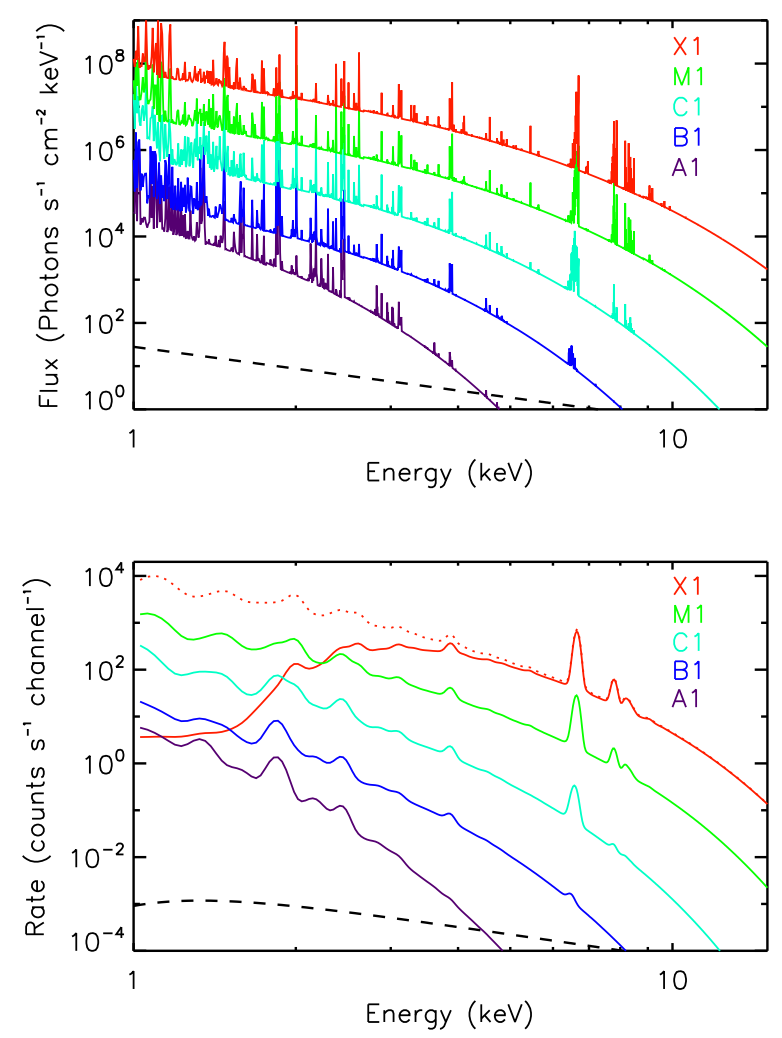

Figure 1. Top: Model spectra for different flare classes computed using CHIANTI. Bottom: Model spectra convolved with the XSM spectral response showing expected observations. Black dashed lines on both plots show the estimated Cosmic X-ray Background spectrum.

Another possible contribution to the background in the XSM is from persistent X-ray sources that are within the FOV of the instrument. However, even for the Crab X-ray source, which is the standard candle in X-ray astronomy and one of the brightest X-ray sources, the expected count rate in the XSM is 0.03 counts $\mathrm{s}^{-1}$, which is negligible in comparison to the CXB background. Apart from the X-ray background, charged particles and secondary emission produced by their interaction with satellite structures can also contribute to the background in the XSM. However, as the XSM detector is protected with package walls from all directions except for the small aperture, this contribution is expected to be of the same order or lower than the CXB background.

In order to determine the nominal range of solar flare classes where the XSM will operate with and without the beryllium filter, the expected count rates are computed from the simulated spectra. The estimated count rates are shown as a function of flare class in Figure 2. Shaded regions represent the range of count rates expected based on the typical range of temperatures and emission measures for each flare class presented in Feldman et al. (1995). The green shaded 


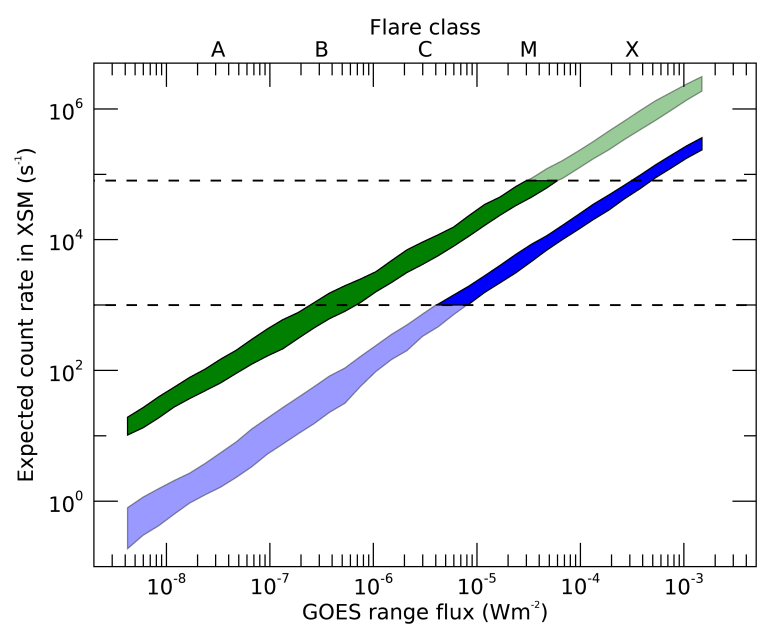

Figure 2. Expected count rate in the XSM for different flare classes. Blue and green shaded regions correspond to count rates with and without the Be filter, respectively. Dark colors represent the expected modes of operation for the threshold rates for filter change shown by the horizontal dashed lines. The XSM operates without the Be filter (shown in dark green) up to a count rate of 80,000 counts $s^{-1}$, beyond which it operates with the Be filter in front of the detector (shown in dark blue) until the count rate drops below 1000 counts s $^{-1}$.

region shows count rates without the thick Be filter, whereas the blue shaded region corresponds to rates when the Be filter is present in front of the detector. Considering that the transition to the Be filter occurs at 80,000 counts $\mathrm{s}^{-1}$ and the transition back to open position is at 1000 counts $\mathrm{s}^{-1}$, the dark blue and dark green regions in the figure represents the nominal operation modes with and without the Be window. These simulations suggest that the transition to the Be filter would occur around the M5 class of solar activity. Also, up to X5 class of solar activity, the count rates stay within $10^{5}$ counts $\mathrm{s}^{-1}$, where no degradation is expected in the spectral performance (Mithun et al., 2020a). It may also be noted that above X5 and up to X9 class, though the spectral resolution would be slightly inferior $(\approx 220 \mathrm{eV})$, the observations would still be useful.

\subsection{Observation Plan and Data Analysis}

Solar observations with the XSM are planned according to the two orbital seasons of the Chandrayaan-2 spacecraft (Mithun et al., 2020a), each spanning approximately three months. During the three months of the 'dawn-dusk' season (D-D), the XSM observes the Sun almost continuously. At the beginning and the end of this season, there are short durations $(\approx 20-30$ minutes per $\approx 120$ minute orbit) of occultation of the Sun by the Moon, which vanishes as the D-D day approaches. During the $\approx 30$ day period around the D-D day, the XSM has almost uninterrupted observations of the Sun. Apart from the occultation period, there can be short periods $(\approx 10$ minutes per orbit, a couple of times a day) of operation of other instruments on the spacecraft during which Sun is out 
Table 2. Approximate duration of orbital seasons of Chandrayaan-2.

\begin{tabular}{ccc}
\hline Season & Period 1 & Period 2 \\
\hline Dawn-Dusk & August 10 - November 20 & February 14 - May 20 \\
Noon-Midnight & November 20 - February 14 & May 20 - August 10 \\
\hline
\end{tabular}

of the field of view of the XSM. During the 'noon-midnight' season (N-M), the $\mathrm{XSM}$ has a much lower cadence of observations. In the initial and last $\approx 20$ days of the season, the attitude definition is such that the Sun is completely out of the field of view of the XSM, and hence no observations are available during this time. After the initial $\approx 20$ days, the Sun enters into the FOV of the XSM with an exposure time of few minutes that increases up to $\approx 25$ minutes per orbit until the N-M day. After the N-M day, exposure per orbit starts decreasing, and the Sun is not in FOV for the last $\approx 20$ days of the season. Table 2 gives the approximate days of the year covered by both the seasons.

Data from all payloads of the Chandrayaan-2 spacecraft are downloaded at the Indian Deep Space Network (IDSN) and the Deep Space Network (DSN) ground stations (Vanitha et al., 2020). After pre-processing at the Indian Space Science Data Center (ISSDC), Bangalore, the XSM level-0 data sets are sent to the Payload Operations Center (POC) located at the Physical Research Laboratory (PRL), Ahmedabad, where the higher-level data processing is carried out. Raw (level-1) and calibrated (level-2) data sets of the XSM, organized into day-wise files, are then archived following the Planetary Data System-4 (PDS4) standards. The XSM data will be made available publicly from the ISRO Space Science Data Archive (ISDA) at ISSDC after a lock-in period of a maximum of nine months after each observing season.

For the analysis of the XSM data, a user-level software named XSM Data Analysis Software (XSMDAS) and the required calibration database (CALDB) will also be made available (Mithun et al., 2020b). XSMDAS consists of individual modules to generate data products with a user-defined set of input parameters. All the data files of the XSM generated by XSMDAS are in FITS format. The raw data include the payload data frames, housekeeping information, and various observation geometry parameters. Using the XSMDAS modules light curves, spectra, and associated response matrices can be generated from the raw data with the required time or energy ranges and bins. The XSM data archive also contains standard calibrated products which are generated by the default processing at the POC. These include a time-series spectrum and total light curve for the full energy range, both with a time bin size of one second.

Spectra and response files generated by XSMDAS are directly usable with standard spectral analysis tools used in X-ray astronomy such as XSPEC (Arnaud, 1996) and ISIS (Houck and Denicola, 2000). The time-series spectrum file which is part of the data archive can also be loaded into OSPEX, which is an IDL-based spectral fitting program in the SolarSoft (SSW) distribution. For this purpose, an IDL routine has been provided as part of the XSMDAS distribution. Detailed descriptions of the XSM Data Analysis Software and algorithms, data 
processing at the POC, and the contents of the XSM data archive are presented in Mithun et al. (2020b).

\section{In-flight Performance and Calibration}

After the launch of Chandrayaan-2, the XSM was first powered-on for a short duration in earth-bound orbit to verify its performance. Similar short observations were carried out in the lunar orbiting phase to investigate any effect of passage through the earth's radiation belt. Regular observations with the XSM started on 12 September 2019, and it has been operating almost continuously since then. The XSM acquires data even when the Sun is not within the FOV in order to obtain background measurements. The instrument is powered off occasionally for short periods during orbit maneuvers and other mission-critical operations.

The performance of the XSM has been evaluated during the six months of inorbit operations with the onboard calibration source, background measurements, as well as solar observations, as discussed in this section. We examine the stability of the energy resolution and gain using calibration source spectra and compare the performance with the ground measurements, and using both background and solar observations, we establish the sensitivity limit of the XSM. Further, with a careful analysis of the quiet Sun observations at different Sun angles, we provide a refinement to the effective area which was obtained from ground calibration alone.

\subsection{Energy Resolution and Gain}

The spectral performance of the XSM is being monitored by using the onboard calibration source mounted on the filter wheel mechanism. Data were acquired with the calibration source during the in-orbit commissioning phase and at regular intervals since. The raw calibration spectra obtained were corrected for gain using the parameters obtained during ground calibration. Lines in the pulse invariant spectra thus obtained were fitted with Gaussians. The spectral resolution, defined by the full width at half maximum (FWHM) of the line at $5.9 \mathrm{keV}$, over time is shown in Figure 3 The peak energy of the same line is also shown as a function of time in the bottom panel of the figure.

It can be seen that the spectral resolution remains the same as that obtained prior to the launch and has remained constant over the six-month duration in-orbit. This shows that the instrument is working flawlessly, and there is no degradation in the performance after the spacecraft's passage through the earth's radiation belts and due to the lunar environment. Line energy as estimated from gain corrected XSM spectra is consistent with the incident energy and shows no variation with time. This confirms that the ground calibration obtained for the gain holds good in space, and there is no variation in the gain parameters so far. Monitoring of the gain and resolution of the XSM will continue with calibration source observations, and the CALDB will be updated in case of any changes in these parameters. 


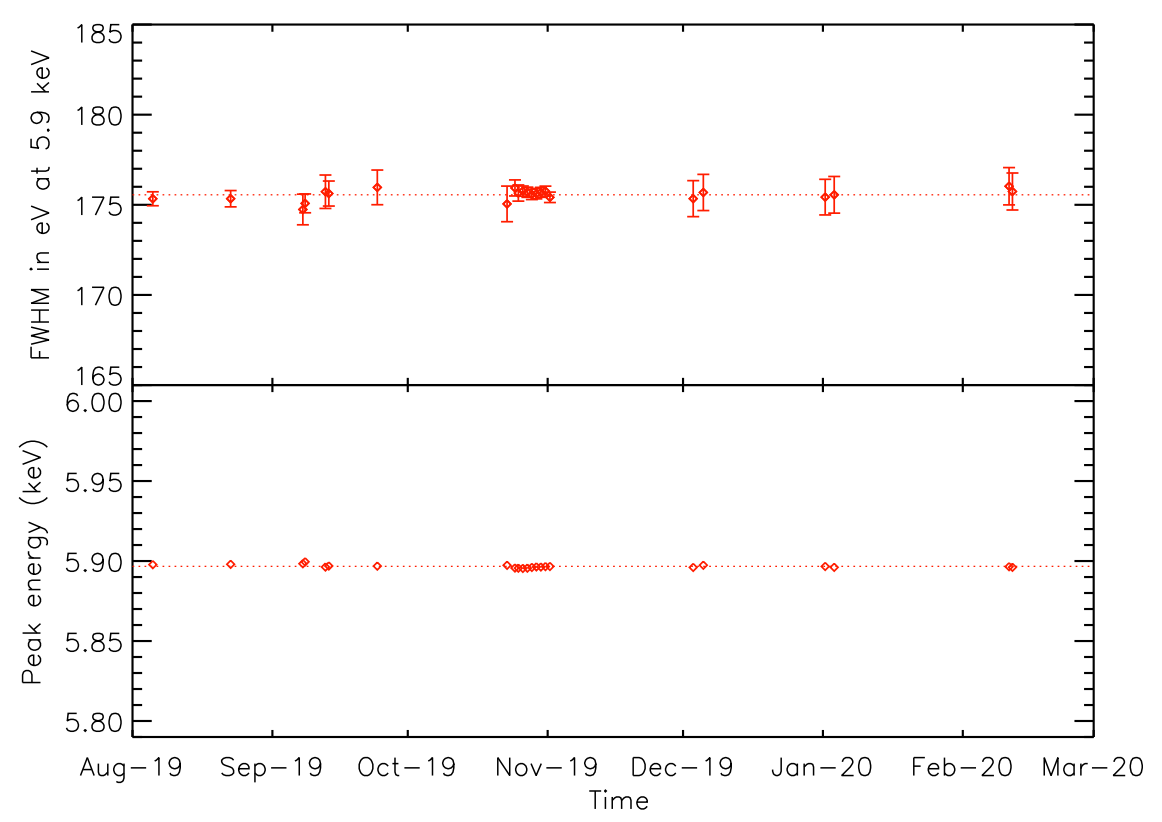

Figure 3. The energy resolution (FWHM) and estimated peak energy for the $5.9 \mathrm{keV}$ line from the onboard calibration source for the first six months of the in-flight operation of the XSM. Dotted lines show the mean values.

\subsection{Background and Sensitivity}

The background rate in the XSM detector primarily determines the sensitivity for solar observations during low solar activity periods. Background measurements are available when the Sun is either occulted by the Moon or is out of the XSM field of view. In the commissioning phase, on 07-08 September 2019, observations of the Sun with the XSM were carried out with intervening durations of occultation. Figure 4 shows the count rate observed with the XSM during this observation showing both the background and the quiet Sun. The observed background rate is $\approx 0.15$ counts s$^{-1}$ very close to the estimated CXB rate of 0.1 counts $\mathrm{s}^{-1}$. The difference is attributed to the additional contribution from particle-induced background. As seen from the figure, the background is approximately 35 times lower than the count rate from the Sun even during this quiet period when the solar activity was well below the A1 level. It may be noted that apart from the $\approx 0.15$ counts $\mathrm{s}^{-1}$ background events, the XSM also records $\approx 1-2$ counts $\mathrm{s}^{-1}$ events that deposit energy greater than the high energy threshold of the XSM which are recorded in the last channel of the instrument that is ignored for all spectral analysis. These events are also due to high energy particle interactions in the detector, but they do not affect the spectral measurements.

During September 30 - October 01 of 2019, two B1 class flares occurred that were also detected by the GOES XRS instrument. Figure 5 shows the XSM 


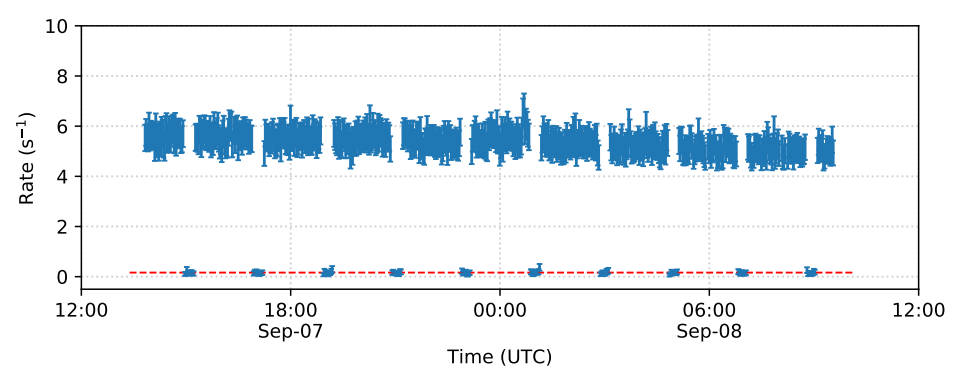

Figure 4. XSM light curve with $100 \mathrm{~s}$ bin size during 07-08 September 2019 with periods of solar observations and occultation by the Moon. The red dashed line shows the mean background count rate during the periods when the Sun was occulted by the Moon, which is $\approx 0.15$ counts $\mathrm{s}^{-1}$. During this period of very low solar activity, counts from the Sun are detected by the XSM well above background.

observations during this period. The top panel shows the observed light curve in 100 second bins, the middle panel shows the flux estimated for the wavelength range of $1-8 \AA$ (the GOES range) by integrating the XSM spectrum within this range, and the bottom panel shows the dynamic spectrum for the same duration. For comparison, the typical background rate is shown in the top panel with a dashed line. It is evident from the plots that even when the solar activity was an order of magnitude below A1 class, the count rates in the XSM, for the Sun, are significantly higher than the background. Spectral variability during the flares is evident from the dynamic spectrum. The integrated spectrum for the duration of the peak of the first B1-flare, marked by the shaded region in Figure 5, is given in Figure 6. For comparison, the background spectrum is also shown. It is to be noted that for low-intensity B1 class flares, spectroscopy at low energies up to $\approx 6 \mathrm{keV}$ is unaffected by the background; however, at higher energies, the background is important to consider. It can be seen that the background spectrum has a line at $\approx 7.5 \mathrm{keV}$, which corresponds to Ni-K $\alpha$. This arises from a thin nickel layer present between the aluminium of the collimator and its silver coating, and the same was observed during ground calibration as well. As the energies of the lines in the solar spectra during large flares (see Figure 1 ) do not coincide with that of this line, it is not expected to interfere with the spectroscopic analysis.

Cosmic X-rays, the primary component contributing to the background in the XSM, are not expected to vary with time. On the other hand, the particleinduced background can be variable. In order to investigate the variation of the background in the XSM, we examine the light curve in the higher energy band above $6 \mathrm{keV}$ over the six months of in-flight operation. As the solar activity was low during this period, at these energies, the contribution from the Sun is expected to be negligible in comparison to the background. Figure 7 shows the XSM light curve above $6 \mathrm{keV}$, where each point is the mean value for the day. The light curve clearly shows a small but distinct variability in the background. In the first part, there is a systematic increase in the daily average count rate, which then has sudden variations in three later instances. The vertical dashed lines in 


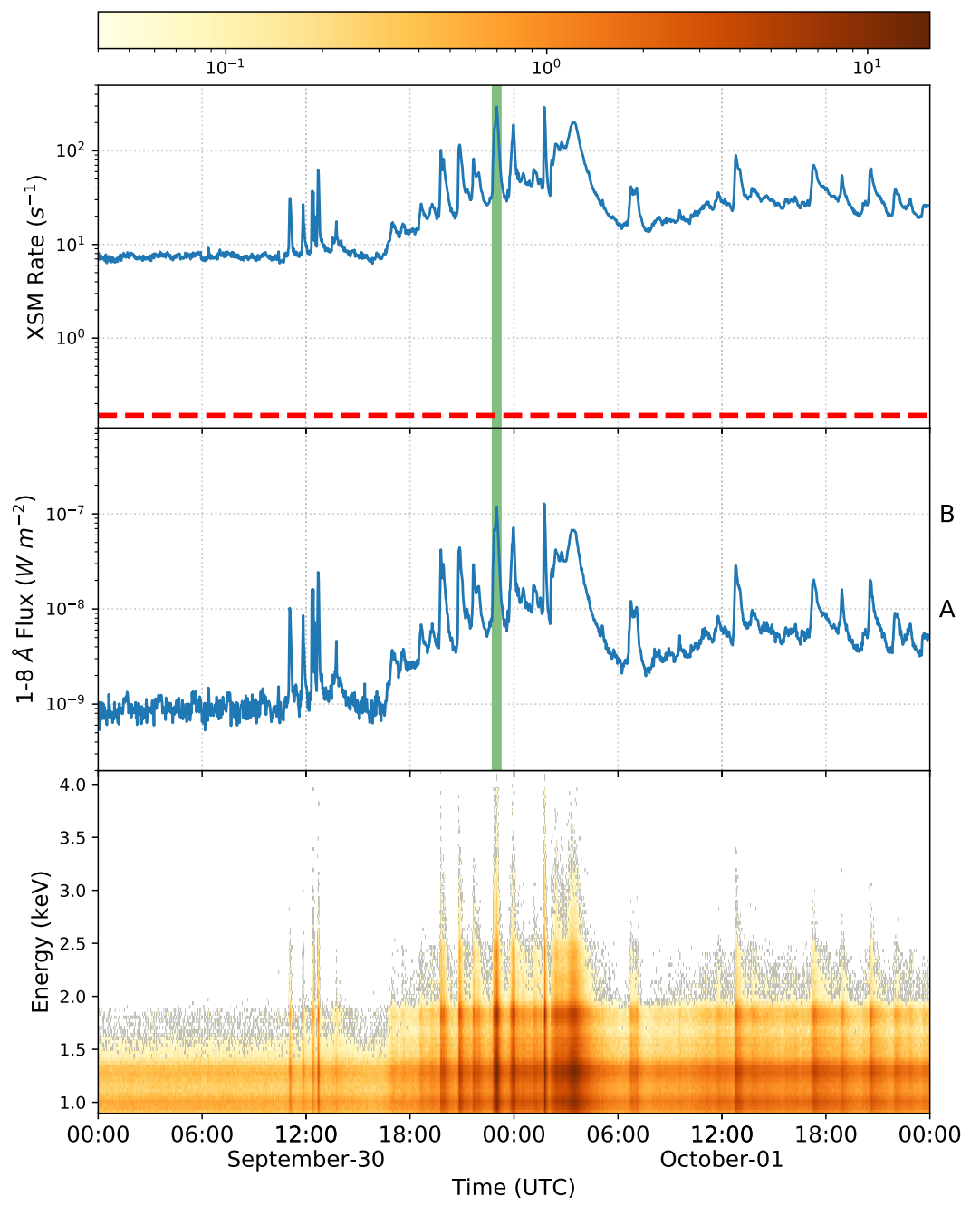

Figure 5. Solar light curve (top), X-ray flux in the 1-8 $\AA$ (1.55-12.4 keV) range estimated from the XSM data (middle), and the dynamic spectrum (bottom) with a time bin of 100s. In the top panel, the red dashed line corresponds to the background count rate. The duration includes periods of very low activity showing a few A-class and sub-A class flares, and two $\mathrm{B}$-class flares. The dynamic spectrum shows the spectral variability during the flares. The integrated spectrum for the shaded duration around the peak of the first B class flare is given in Figure 6

the figure represent the times when the attitude configuration of the spacecraft was changed that coincide with the sudden changes in the count rates. Hence, it is understood that there is a correlation between the attitude configuration and the background in the XSM. There may also be further variations within a day that are averaged out in this plot. There are also multiple short periods with enhanced background rates that coincide with the passage of the spacecraft through the magnetospheric tail of earth, marked by the gray shaded regions in 


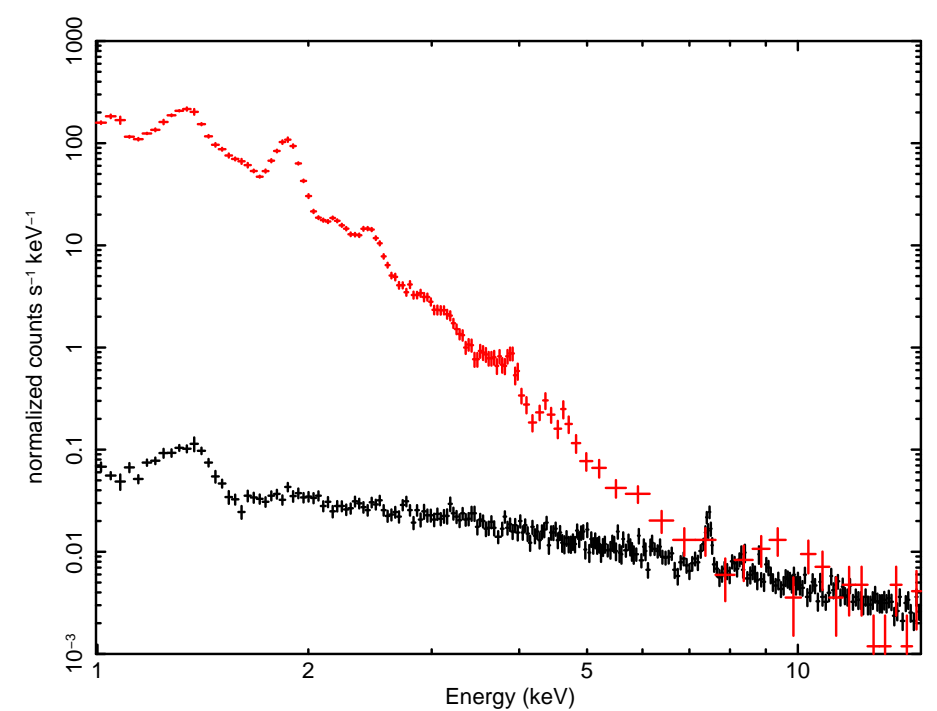

Figure 6. Solar spectrum (red) as measured by the XSM during the B1 class flare on 30 September 2019 for the shaded duration (1700 s) shown in Figure 5. A representative background spectrum (black) of the XSM is also shown for comparison. The line seen in the background spectrum at $\approx 7.5 \mathrm{keV}$ is of instrumental origin as mentioned in the text.

the figure. An increase in the background in these durations is expected from the enhanced particle densities in the geo-tail (Narendranath et al., 2014b). It may be noted that the magnitude of variations in the background is very small compared to the solar flare spectrum, and hence it will not affect the spectral analysis of solar flares, which is the primary goal of the XSM. However, in order to extend the spectral analysis of the quiet Sun to higher energies, the background variations need to be understood and modeled, which is planned to be carried out in the near future.

Based on the in-flight observations of the background as well as the Sun, it can be seen that the XSM is sensitive enough to measure solar activity in the soft X-ray band at flux levels of at least two orders of magnitude lower than A1 class events. The XSM is capable of detecting flare events that fall below A1 class and also provides their spectral measurements. The XSM has observed many such flares, and the results will be reported in a future publication.

\subsection{Collimator Response and Effective Area}

The effective area of the XSM is critically dependent on the collimator response and parameters, such as the thickness of the window, detector thickness, and dead layer. The collimator response was experimentally determined for estimating the effective area, whereas, for the other parameters, which are internal to the detector module, the manufacturer provided values were used (Mithun et al., 


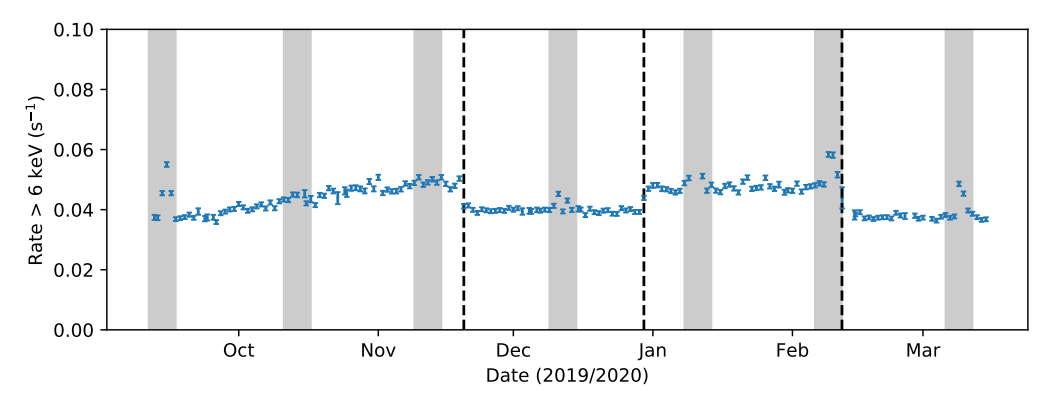

Figure 7. The daily average XSM count rate above $6 \mathrm{keV}$ plotted as a function of time. The variability seen is associated with background variations. Vertical dashed lines show the instances of change in attitude configuration of the spacecraft, which coincides with sharp changes in the count rate. Gray shaded regions correspond to the passage of the spacecraft through the earth's magnetospheric tail, where enhanced particle concentrations are expected.

2020a). Here, we examine the adequacy of the ground calibration estimate of the effective area using the in-flight observations. In this section, we discuss the investigation of whether the field of view of the XSM is symmetric as understood from the ground calibration experiment and the re-calibration of the effective area with observations of the quiet Sun.

\subsubsection{Field of View}

When the Sun is at least partially within the field of view of the XSM, the recorded count rates are significantly higher than the background rate of $\approx 0.15$ counts $\mathrm{s}^{-1}$. So, the XSM count rate can be used to verify whether the Sun is within the field of view or not, thus identifying the null points, i.e., the edge of the FOV of the XSM. We used all available observations until March 2020 for this purpose. Light curves with a bin size of 10 seconds were generated for the entire period, ignoring the durations when the Sun was occulted by the Moon. For each time bin, average polar $(\theta)$ and azimuthal $(\phi)$ angles of the Sun with respect to the XSM instrument frame were computed. Figure 8 shows the polar plot of the position of the Sun during each time bin, where the radial axis represents the polar Sun angle. The time bins having count rates $5 \sigma$ higher than the background rate are shown with blue points, and the others are shown with black points. The solid orange circle shows the edge of the FOV as obtained from the ground calibration experiment. It can be seen that the blue points representing the presence of Sun in the FOV are all within the orange circle, which shows that the onboard observations are consistent with a symmetrical field of view as measured on the ground. It also demonstrates that the estimates of the Sun angle are correct. This is important to ensure because the Sun angle is used in estimating the effective area for a given observation. 


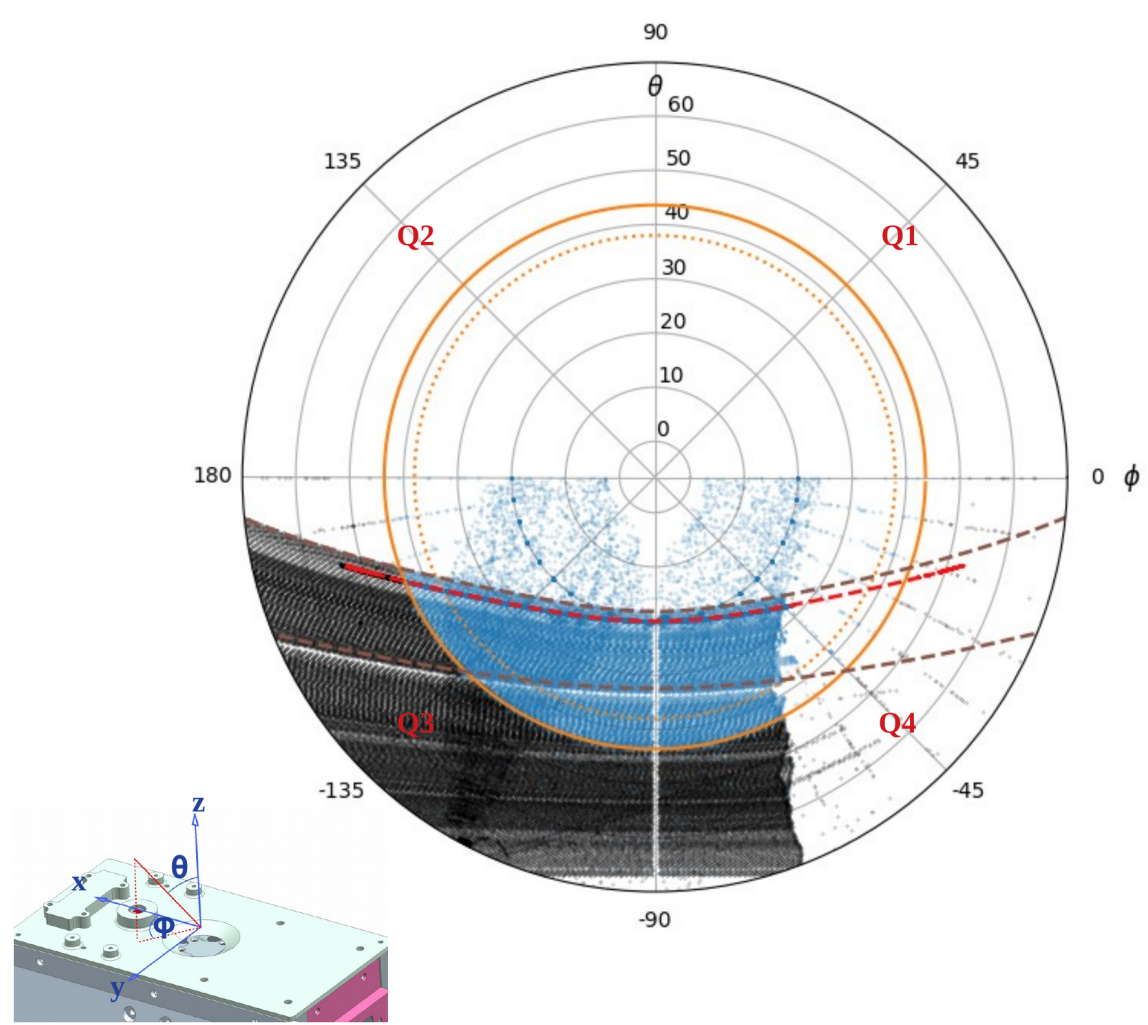

Figure 8. Polar plot showing the position of the Sun in the XSM reference frame and the corresponding count rate. Each point in the plot corresponds to the position of the Sun during a time bin of $10 \mathrm{~s}$, and its color represents the count rate observed by the XSM. Time bins with count rates at $5 \sigma$ higher than the background rate are shown in blue, and others are in black. The definition of the XSM reference frame is shown in the schematic at the bottom left corner of the figure. The center of the polar plot corresponds to the XSM boresight with polar angle $\theta=0$ and the radial axis represents $\theta$ values up to $70^{\circ}$. The azimuthal angle $(\phi)$ is defined in the range from $-180^{\circ}$ to $+180^{\circ}$. It may be noted that due to the mounting geometry of the XSM and the specific spacraft attitude configuration, all observations have $\phi$ within a range of $-180^{\circ}$ to $0^{\circ}$ (quadrants 3 and 4) and mostly have $\theta>20^{\circ}$. The red dashed line shows the track of the Sun within the XSM FOV during nominal D-D season observations, and the brown dashed-lines show parts of the track during two representative days in the N-M season. Durations of the occultation of the Sun, which happens only in quadrant 4, are not included in this plot, which results in the asymmetry between quadrants 3 and 4 . The solid orange circle represents the null points of the XSM FOV as obtained from ground calibration, and the dotted orange circle represents the full FOV. It can be seen that the blue points lie within the FOV, which shows that the FOV as determined from ground calibration is consistent with the onboard solar observations (see text for further details).

\subsubsection{Effective Area Calibration}

Generally, X-ray spectrometers utilize observations of the standard source Crab for in-flight calibration of effective area, but this is not feasible for the XSM due to its very small aperture area and large field of view. Hence, we have to rely on the quiescent Sun observations when the inherent source variability is 
minimum. As the angle between the Sun and the XSM bore-sight varies within each orbit during nominal observations, that data can be used to characterize the effective area as a function of angle, avoiding any requirement of separate calibration observations.

As the angle between the Sun and the XSM boresight varies with time, the raw light curve shows modulation; however, these modulations are expected to get corrected after considering the corresponding effective area as determined from the ground calibration. The top panel of Figure 9 shows the raw (gray) and effective area corrected (blue) light curves for the observation of on 17 September 2019 when the Sun was quiet and showing low X-ray variability. The other two panels of the figure show the polar $(\theta)$ and azimuthal $(\phi)$ Sun angles during the same period. It can be seen that even after incorporating the effective area correction based on the ground calibration, the modulation in the light curve over the orbital phase where $\phi<-90^{\circ}$ is not fully corrected. A possible reason for this could be asymmetry in the FOV; however, this is ruled out based on the analysis presented in the previous section. Thus, the uncorrected modulation over half of the orbital phase shows that the actual effective area has an azimuthal angle dependence and suggests that the effective area obtained from ground calibration needs further refinement.

For this purpose, we considered all the available observations during the 'dawn-dusk' seasons up to March 2020 and identified the durations when the variability in solar flux was minimum. This was done by examining the light curves manually and ignoring durations when even very small flares or any other variability within a day's timescale were present. Finally, observations from 42 days were selected for the analysis. It may be noted that for all these observations which are in the 'dawn-dusk' season, the Sun follows the same track in the XSM reference frame, which is shown by the red dashed line in Figure 8 .

From data for each day, we generated light curves in the $1-15 \mathrm{keV}$ energy range, scaled by the effective area from ground calibration, with a time bin size of $10 \mathrm{~s}$, and computed the corresponding mean polar $(\theta)$ and azimuthal $(\phi)$ Sun angles. To investigate the observed asymmetry in the effective correction with orbital phase, we estimated seperately, the mean count rate as a function of $\theta$ for $\phi<-90^{\circ}$ (in quadrant 3 of the XSM reference frame, hereafter Q3) and $\phi>-90^{\circ}$ (in quadrant 4 of the XSM reference frame, hereafter Q4) separately. In order to remove any incident flux variations between different days of observation, we scaled the count rates for different $\theta$ with the count rate at $\theta=20^{\circ}$ for each day. These day-wise normalized count rates for each $\theta$ were averaged to obtain the results shown in Figure 10 top panel with blue star (Q3) and open square (Q4) data points. It can be seen that for Q4, the normalized count rate, corrected with the effective area from ground calibration, remains constant with angle, whereas in Q3, it decreases with increasing angle. This shows that the estimate of the effective area reduction with $\theta$ from ground calibration is correct for Q4, but for Q3 it is steeper than the ground estimate.

This enhanced reduction in effective area for observations in Q3 could be due to a difference in collimator response, in which case it would be independent of energy, or due to difference in window transmission, in which case it would be energy dependent. In order to probe whether it is dependent on energy or not, we 


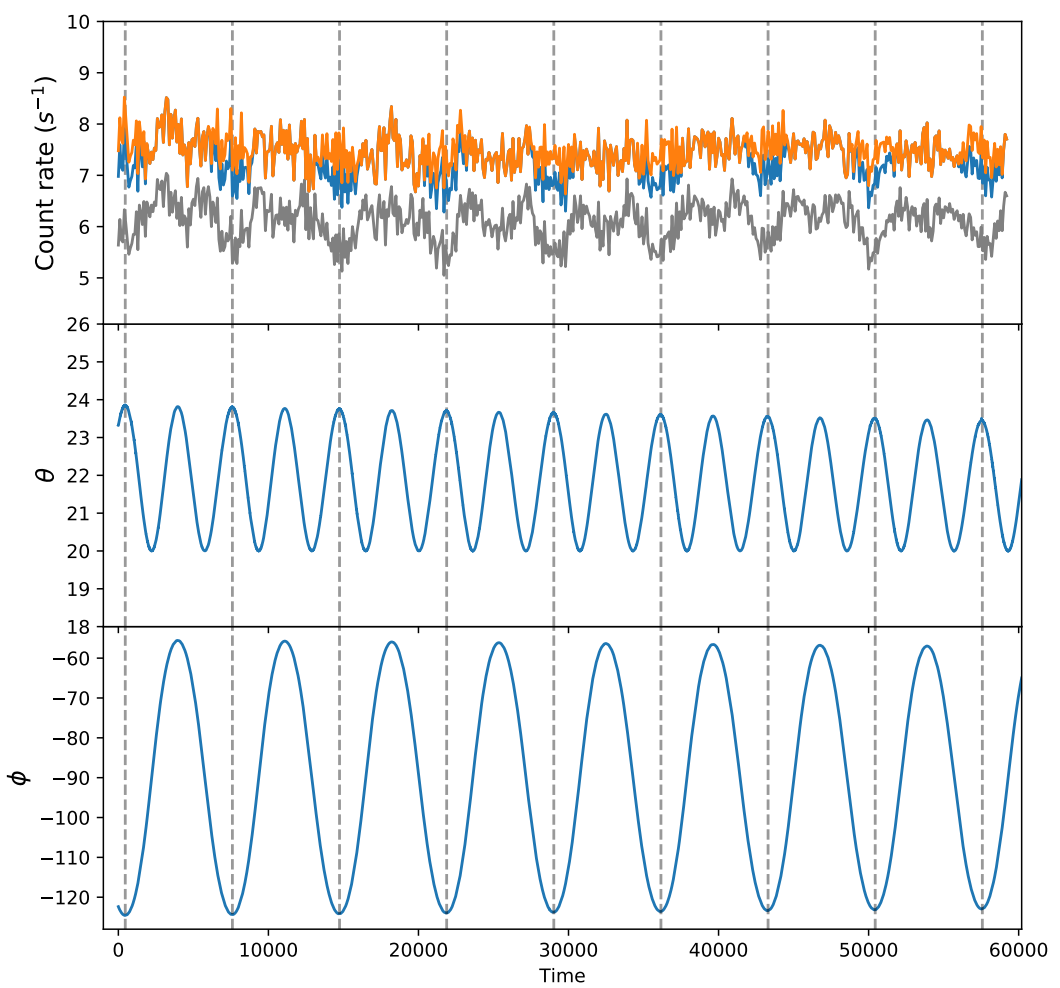

Figure 9. Light curves obtained with the XSM on 17 September 2019 when the Sun was quiet without much inherent variability in the X-ray flux are shown in the top panel. The raw light curve (gray), the light curve corrected with ground calibration effective area (blue), and that corrected with the updated effective area (orange) are shown. Note that the mean value of the effective area corrected light curves are higher than the raw light curve as they are scaled to provide count rates corresponding to on-axis observations with the XSM. The $\operatorname{polar}(\theta)$ and azimuthal $(\phi)$ Sun angles are shown in the middle and bottom panels. The raw light curve shows significant modulation during the minima of the azimuthal angles marked by the vertical dashed lines in the figure, which has not been corrected by the effective area from ground calibration. With the refined effective area correction, the modulations in the light curve have been almost completely removed.

used light curves in two energy ranges: $1.0-1.2 \mathrm{keV}$ and $1.2-1.5 \mathrm{keV}$. From these light curves for each day of observation, count rates were estimated as a function of $\theta$ for both quadrants, and a ratio of rates in the lower energy band to the higher energy band was computed for each $\theta$. The count rate ratio as a function of angle for each day was normalized to that at $\theta=20^{\circ}$ and were averaged to obtain the result shown in the middle panel of Figure 10 . If the reduction in the effective area were due to an energy-independent factor, this ratio is expected to remain constant with angle. It can be seen from the figure that for Q3, the ratio of count rates does not remain constant with angle, suggesting that the reduction in effective area from ground calibration estimate is energy dependent. As the 

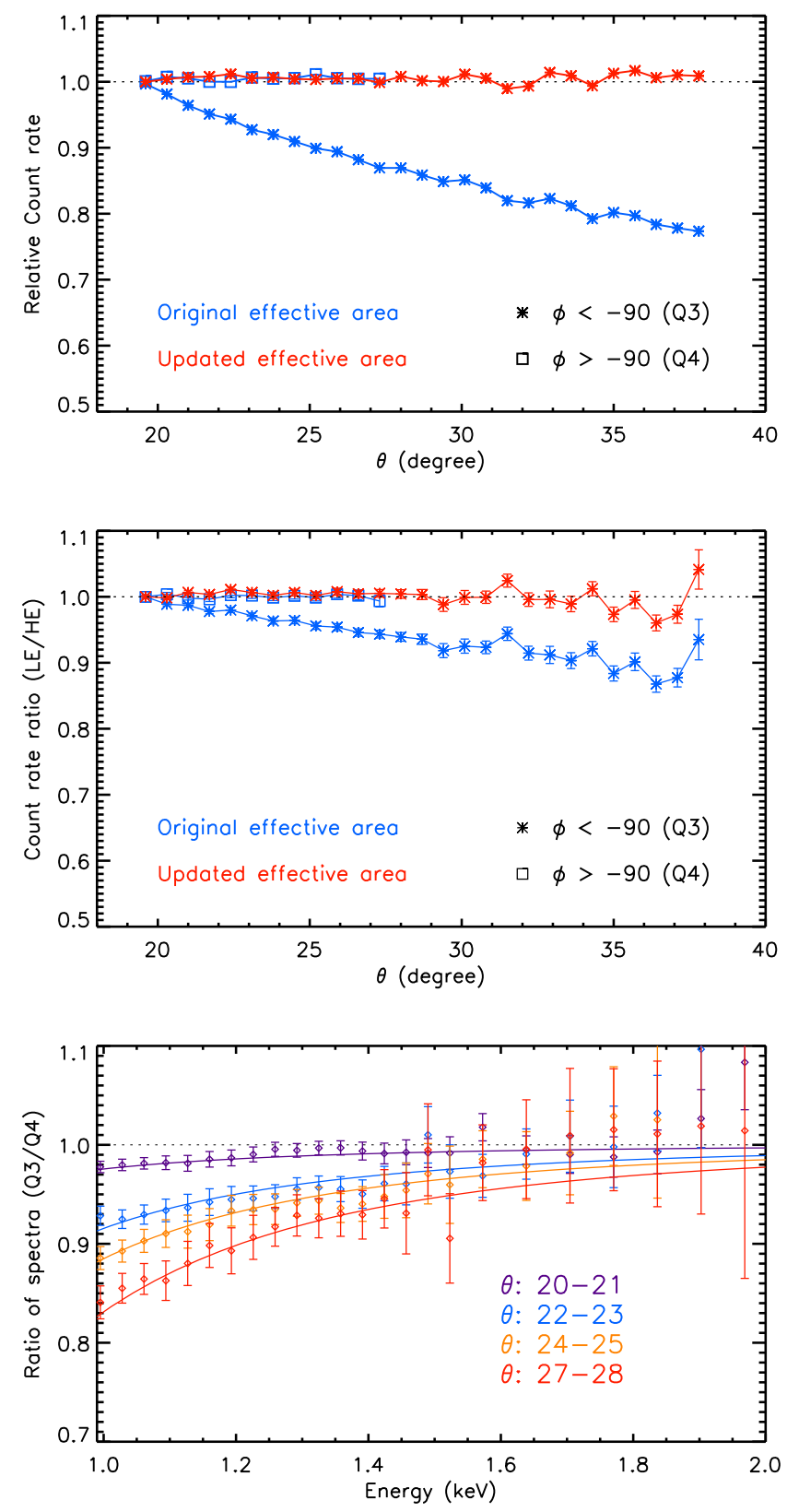

Figure 10. Top panel: Mean XSM count rates during quiet Sun observations normalized to that at $\theta=20^{\circ}$ as a function of polar angle $(\theta)$, for observations with $\phi<-90^{\circ}$ (Q3) and $\phi>-90^{\circ}(\mathrm{Q} 4)$. In the case of $\mathrm{Q} 4$, the count rates corrected with the original effective area (blue open square) remain constant with angle confirming that the effective area is valid, whereas the count rates show a decreasing trend with angle for observations in Q3 (blue star). Middle panel: Normalized ratio of count rates in the energy ranges 1-1.2 keV (Low energy or LE) to $1.2-1.5 \mathrm{keV}$ (High energy or HE) as a function of $\theta$. The ratio shows a monotonic decrease with the angle in the case of Q3 (blue star), suggesting that the correction required in the effective area is energy dependent. Bottom panel: Ratio of spectra for observations in Q3 to that in Q4 for different bins of $\theta$. Solid lines show the additional absorption required for observations in Q3 to explain the observed deviation of spectral ratio from unity at lower energies. This correction factor is incorporated in the XSM effective area (for Q3), and the resultant corrected count rates and the ratio of count rates in two energy bands are shown with red points in the top and middle panels, respectively. The count rates and the ratio remains constant with $\theta$ which demonstrates the efficacy of the correction in the effective area 
ratio is less than unity and decreases with the increase in angle, the effective area for Q3 seems to require an additional absorption factor that depends on angle.

To quantify this additional energy and angle dependent absorption factor in effective area, we used the available spectral information. From the same data selected for the earlier exercise, spectra were generated in each one degree bin of $\theta$ for the two quadrants separately. Using them, ratios of spectra when the Sun is in Q3 to that in Q4 were computed for each $\theta$ bin for all days. These day-wise spectral ratios were averaged to obtain the final set of ratios. As observations in Q4 are limited to a $\theta$ of $28^{\circ}$, the ratios are available for $\theta$ bins up to $28^{\circ}$ only. A representative set of spectral ratios are shown in Figure 10 bottom panel. It is evident from the figure that fewer counts are detected at lower energies for observations in Q3 compared to Q4, and this deviation increases with angle, confirming the presence of an additional absorption in this quadrant.

The exact origin of such absorption in only one region of the field of view is not yet clear. It probably arises from the detector module, maybe due to the varying thickness of the Be window. However, it is difficult to derive any conclusions based on the available data. Hence, we determine this additional absorption factor for Q3 empirically from the observed spectral ratios by modeling it as absorption by beryllium. Solid lines overplotted on the observed spectral ratios in the bottom panel of Figure 10 shows the empirically determined effective area correction terms that correspond to absorption by additional beryllium whose thickness ranges from $\approx 0.2 \mu \mathrm{m}$ to $\approx 1.6 \mu \mathrm{m}$ for different angles. It can be seen that the derived effective area correction matches the observations very well. As the spectral ratios are available only up to $28^{\circ}$, the correction terms for the effective area at higher angles were obtained by extrapolating the dependence on $\theta$. We then incorporate this effective area correction term in CALDB and generate light curves corrected for the updated effective area. Count rates and the ratio of count rates in two energy bands as a function of $\theta$ after scaling with this updated effective area are shown in red color on the top and middle panels of the Figure 10, respectively. The count rate and the ratio are now constant with the polar angle showing the efficacy of the effective correction term obtained. The light curve with the updated effective area for the data of 17 September examined earlier and shown in Figure 9 also demonstrates the same.

Apart from the beryllium window thickness, another factor that would cause uncertainties in the effective area is the thickness of the silicon dead layer in front of the detector. The dead layer affects the effective area the most at energies just above the Si K edge around $1.84 \mathrm{keV}$, which in turn, may cause uncertainties in the measurement of solar abundances of Si. It can be seen from Figure 10 that area uncorrected spectral ratios do not have any significant deviations from unity at those energies, and hence it can be concluded that the dead layer thickness is not varying significantly over the detector area. However, the absolute value provided by the manufacturer $\left(100 \mu \mathrm{m} \mathrm{Si}\right.$ and $\left.80 \mu \mathrm{m} \mathrm{SiO}_{2}\right)$ may have uncertainties. To estimate its effect on the $\mathrm{Si}$ abundance measurements, we simulated spectra assuming different dead layer thicknesses ranging from zero to double the expected value. These were further fitted with the standard response with the manufacturer provided values of dead layer thickness to obtain 
measurements of Si abundances and all other parameters. We find that, even with the assumption of extreme ranges of dead layer thickness, the abundances measured for $\mathrm{Si}$ are within $\pm 0.5 \%$ of the actual value, which is very small compared to the typical statistical errors associated with abundance measurements from the XSM spectra. Further, as the effective area near line complexes of other elements are significantly less affected by the dead layer thickness, their abundance measurements will be unaffected. Hence, we conclude that the effect of uncertainties on the dead layer thickness can be safely ignored.

In order to estimate the overall uncertainties in the relative effective area with the polar angle, the area corrected count rates in different angle bins shown in the top panel of Figure 10 were used. The standard deviation of the count rates is $\approx 0.8 \%$ and hence we quote a conservative limit of $1 \%$ uncertainty in the relative effective area with angle. It may be noted that the present analysis was carried out with D-D observations, and thus we have a fairly robust estimate of the effective area for these ranges of $\theta$ and $\phi$. For the N-M case, this would serve as a starting point, but we plan to continue the investigation of $\phi$ dependence of effective area as a function of energy as more data are acquired in this attitude configuration. Further, during the XSM operation so far, the Sun has been extremely quiet with emission well below A-class for most of the time, thereby yielding very low count rates mostly limited up to $2 \mathrm{keV}$. These observations were used for the present analysis, and the absorption factors were estimated with the spectral ratios in the $1-2 \mathrm{keV}$ band. Although these observations seem to suggest that the ratios flatten above $\approx 1.6 \mathrm{keV}$ or so, this is limited by statistics. Observations with higher count rate extending to higher energies, when the Sun becomes more active, are likely to provide further insights into this. Another point to note is that the requirement of additional correction factor in the effective area concern only the observations when the Sun in Q3. In Q4, the effective area is well understood, and the in-flight observations are consistent with ground calibration estimates. So, it would be good to verify the results for observations in both quadrants separately whenever feasible. In any case, we plan to continue the investigations as and when additional observations become available and the updated effective area will be made available in the revised calibration database.

\subsubsection{Low Energy Threshold}

The low energy threshold below which the XSM does not detect photons is determined by a threshold pulse height setting in the readout electronics, which can be changed by ground command. This ensures that the instrument does not record events due to very small fluctuations present in the signal. After the initial commissioning phase, the default value of the XSM threshold was set to a value that nominally corresponds to $\approx 900 \mathrm{eV}$. However, the spectrum would not have a sharp cut-off at this energy; rather, the detection probability starts to increase from zero at energies below $900 \mathrm{eV}$ and then reaches unity at some energy higher than $900 \mathrm{eV}$. An error function can generally describe this feature near the low energy threshold (e.g., Prigozhin et al., 2016). 


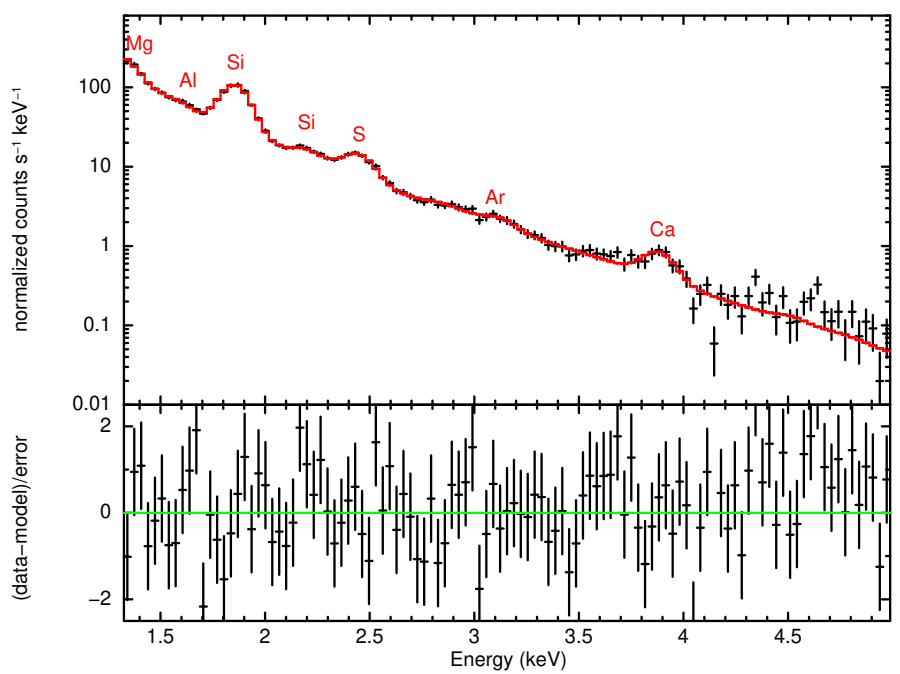

Figure 11. Solar spectrum as measured by the XSM during the B1 class flare on 30 September 2019 shown in the shaded time interval in Figure 6 fitted with vapec model in XSPEC. Spectrum in the energy range of $1.3-5.0 \mathrm{keV}$ is considered for the fitting. The best fit model for a temperature of 6.45 MK is shown in red and the residuals are shown in the lower panel. Elemental line features are marked in the spectrum.

On careful analysis of the solar spectra, after incorporating the corrections for the effective area as described in the previous section, it has been noticed that the effect of the low energy threshold extends up to about $1.30 \mathrm{keV}$. Hence, the predicted counts from response in channels up to this energy would be higher than the actual observation. After the completion of the second D-D observing season, the low energy threshold setting was optimized to bring the effects of the electronic threshold to energies lower than $1 \mathrm{keV}$ while avoiding any spurious events, and the default setting was changed from June 2020. Thus, for observations before June 2020, it is recommended that the spectrum below $1.3 \mathrm{keV}$ is ignored during fitting.

To demonstrate the adequacy of the calibration status of the XSM, a representative flare spectrum on 30 September 2019 (same as in Figure 6) was fitted with a model for isothermal plasma emission. The model in XSPEC named vapec, which computes the X-ray spectrum with the AtomDB atomic database, was used for this purpose. Figure 11 shows the spectrum, fitted model, and the residuals, where the best fit model corresponds to a plasma temperature of 6.45 MK. It can be seen that the model convolved with the XSM response fits the observed data very well. Detailed spectral analysis and interpretations of solar observations with the XSM making use of an XSPEC model that uses the CHIANTI atomic database will be presented in future.

The XSM has been in orbit and operational for nearly one year at the time of writing. Its spectral performance has undergone no variation during this time 
and matches very well with the ground calibration. Gain corrections, taking into account the variations with electronics temperature as well as the X-ray interaction position in the detector, provides energy measurements within an accuracy of $10 \mathrm{eV}$. The observed background count rates are well within the expectations, and hence the XSM is sensitive enough to measure variability in $\mathrm{X}$-ray flux even at the sub-A class level of solar activity. The effective area as a function of angle obtained from ground calibration is refined with the analysis of in-flight data, and the resultant effective area is shown to have an uncertainty of less than $1 \%$; hence the error in flux measurements with the XSM will be of the same order. We plan to validate this further using more observations. We will also continue to monitor the calibration parameters of the XSM like gain and spectral resolution for any changes.

\section{Science Prospects}

The primary objective of the XSM is to aid the measurement of lunar surface elemental abundances with the CLASS instrument. This requires observations during periods of increased solar activity such that fluorescence emission from the lunar surface is significant. A preliminary result of elemental abundance estimates during one low-intensity flare is presented in Narendranath et al. (2020). Observations over the entire mission life are expected to provide abundance measurements over substantial regions of the lunar surface.

Apart from this, the broadband X-ray spectral observations with the XSM with moderately high resolution and unprecedented time cadence will enable one to explore specific science problems in solar physics. Some of the areas where observations with the XSM can contribute are discussed below.

\subsection{Microflares}

Recent observations of the Sun with sensitive instruments like NuSTAR (Wright et al., 2017) and FOXSI-2 (Athiray et al., 2020) have generated considerable interest in microflares. With its high sensitivity, NuSTAR has been able to detect the weakest (thermal energy of $1.1 \times 10^{26} \mathrm{erg}$ ) active region microflare so far (Cooper et al., 2020). Energetically, events classified as microflares are about a million times weaker events than standard flares. Shimizu (1995) had done the first number distribution study of such events using the Yohkoh Soft $\mathrm{X}$-ray telescope and had found that a single frequency distribution with a powerlaw index $(\alpha)$ between 1.5 and 1.6 can explain the occurrence frequency of flares and microflares together, up to an energy of $10^{27} \mathrm{erg}$. It is believed that such micro-flares, or nanoflares with even lower total energy, can deposit a significant amount of energy in the solar corona. However, in order to explain the coronal heating entirely as due to the micro/nano flares, it is required to have a steeper power-law index at lower energies (Hudson, 1991).

As shown in the previous section, the XSM is quite capable of detecting sub-A class flare events having energies of the order of $10^{27} \mathrm{erg}$ and lower.

As the XSM unlike NuSTAR and FOXSI, lacks imaging capabilities the intensity of faintest detectable flares are limited to the background quiescent coronal 
emission. However, the availability of continuous observations over long periods of time and the lower energy threshold extending down to $1 \mathrm{keV}$ make the XSM observations very useful and valuable. Particularly since the XSM is observing during the solar minimum conditions it would be feasible to detect fainter events and provide better constraints on the power-law index for the frequency distribution of microflares. Such measurements will help us in estimating the nature of the frequency distribution of even weaker nanoflares, and thus may be able to shed light on their contribution to the coronal heating.

Microflares are often termed as Active Region Transient Brightenings (ARTB) (Gupta, Sarkar, and Tripathi, 2018). During their evolution phase, they are often seen in ultraviolet bands, although they are undetectable in GOES (MitraKraev and Del Zanna, 2019). Simultaneously observing such events with the XSM along with the Atmospheric Imaging Assembly (AIA) on board the Solar Dynamics Observatory (SDO) will help us to understand their multithermal nature. The SphinX X-ray spectrometer that operated for about nine months in 2009 detected several such events but did not have the UV context images from the SDO available as will be the case of observations with the XSM. However, since the XSM observes the Sun as a star, one needs to make sure that at any instant, there has to be only one such event on the solar disk.

\subsection{Non-thermal Emission in Flares and the Quiet Sun}

Non-thermal processes are dominant during the evolution of solar flares and contribute significantly to the X-ray emission (Saint-Hilaire and Benz, 2005, Krucker et al., 2008, Dudík et al., 2017). A comparison of the observed X-ray spectrum with the model thermal spectrum convolved with the instrument response would indicate whether a non-thermal component is present (e.g., Joshi et al., 2013 . Kushwaha et al., 2014). A time-resolved analysis of XSM spectra during flares would provide insights into the evolution of the non-thermal component. Because of its better sensitivity and much higher spectral resolution compared to previous instruments, measurements with the XSM would especially be useful in probing non-thermal emission during low-intensity flares where the transition from thermal to non-thermal may occur at much lower energies $(\leq 10 \mathrm{keV})$. Joint observations using the XSM and hard X-ray solar spectrometers such as STIX (Krucker, S. et al., 2020) on board the recent Solar Orbiter mission and HEL1OS (Sankarasubramanian et al., 2017) on board the upcoming Indian mission Aditya-L1 will also be very useful. Joint fits to the spectra over a wider energy band combining the XSM and STIX/HEL1OS data will allow one to constrain the thermal and non-thermal components better.

In many cases, eruptive flares exhibit pre-flare activity, which is characterized in terms of the duration and intensity of soft X-ray flux prior to the impulsive rise of the flare emission (Fárník, Hudson, and Watanabe, 1996 Chifor et al., 2007; Joshi et al., 2011; Mitra and Joshi, 2019). The X-ray flare light curves indicate that the pre-flare activity comprises of single or closely spaced multiple episodes of energy release (e.g., Joshi et al., 2016; Mitra, Joshi, and Prasad, 2020). Despite observational limitations, RHESSI observations have detected weak yet clear non-thermal component during the pre-flare phase (e.g., Hernandez-Perez et al., 
2019 Sahu et al., 2020) which points toward the role of small-scale magnetic reconnection in destabilizing the magnetic field configuration of the active region and thereby triggering subsequent eruptive flares. Such case studies are very limited in number and, therefore, statistically viable results cannot be inferred from them. Given the better spectral resolution in the energy band of 1-15 keV, XSM data would provide an excellent opportunity to explore the pre-flare activity. The synthesis of XSM measurements with imaging data at multi-wavelengths (such as EUV, UV, and $\mathrm{H} \alpha$ ) during the pre-flare activity would shed light on the triggering mechanisms of solar eruptions.

Non-thermal emission from the quiet Sun corona is considered as an indicator of the presence of nanoflare heated plasma. A careful spectral analysis of the XSM observations of the quiet Sun integrated over long periods can be used to probe this. As the XSM has observed the Sun during the solar minima, there are significant durations without any active regions present on the Sun allowing the spectral study of the quiescent corona even with disk integrated spectra. Of course, such an analysis would require a better understanding of the background spectrum.

\subsection{Overall Temperature Structure of the Quiet and Active Corona}

As different regions of the solar corona demonstrate different temperature structure, it is customary to determine the Differential Emission Measure (DEM) of the whole-disk or that of a specific region, such as the active region or the coronal hole (Landi and Chiuderi Drago, 2008; Mackovjak, Dzifčáková, and Dudík, 2014: Schonfeld et al., 2017). The DEM is derived from the observed intensities and theoretical emissivities of spectral lines. For the quiet Sun, such DEM is seen to peak around $\log \mathrm{T}=6.0$, while for active regions, the DEM peaks around $\log$ $\mathrm{T}=6.2$ (Brosius et al., 1996). Usually, while generating the DEM, most of the lines used are from Extreme Ultraviolet (EUV), and hence, high-temperature regions of the DEM curves are not well constrained. Observations and numerical simulations show that nanoflares can heat the local solar plasma to temperatures of $10 \mathrm{MK}$ (Reale et al.,2009, Allred, Daw, and Brosius, 2018), suggesting that the DEM curves of a nanoflare heated region should extend to similar temperatures. To understand the slope of the DEM curve at this high-temperature, DEM analysis of spectra at X-ray energies is required, which can be attempted using XSM observations. However, in contrast to the EUV observations, DEM analysis with broad-band spectrometers like the XSM would have to take into account the intensity in each energy band due to both line and continuum emission.

\subsection{Understanding Elemental Abundances of the Solar Corona}

It is well known that elements with low $(<10 \mathrm{eV})$ first ionization potential (FIP) are generally at least twice more abundant in the corona than in the photosphere. This phenomenon is known as the FIP effect (Laming, 2015. Del Zanna and Mason, 2018). Low FIP elements are generally ionized in the chromosphere, while the high FIP elements remain at least partially neutral. Several models invoke various mechanisms such as force due to magneto-hydrodynamic waves 
for separation of ions from neutrals, to explain the observed enhancement of low FIP elements (see Laming, 2015 for a recent review). During flaring activity, abundances in the corona are also found to change abruptly. X-ray spectroscopy of the Sun can help us understand such behavior of elemental abundances more accurately. There have been spectroscopic observations which suggest that the mean abundances of some of the elements reach photospheric values during flares (Sylwester et al., 2014, 2015). With high time cadence observations, it would be possible to understand the time evolution of abundances during flaring activity. Broadband X-ray spectral measurements with the XSM in the $1-15$ $\mathrm{keV}$ range covers multiple strong lines of many of the elements present in the corona. By fitting the observed spectra with theoretical models which makes use of atomic databases like CHIANTI, abundances of these elements can be estimated. It is expected that observations with the XSM can constrain the abundances of some of the elements in the quiet corona and active regions and also their evolution during different classes of solar flares.

\subsection{Long Term X-ray Flux Monitoring}

Solar X-ray flux has been continuously monitored by the GOES series of satellites over the past four decades. X-ray flux measurements from GOES have been used as indicators of the solar activity over its eleven-year cycles (e.g., Joshi and Joshi, 2004 Joshi et al., 2015), apart from other measurements, such as sunspot number and magnetic field strength. In recent times, it has been shown that the solar activity is declining based on a steady decrease in solar photospheric fields starting from around 1995 (Janardhan et al., 2015a) and several other signatures (Janardhan et al., 2011, 2018, Sasikumar Raja et al., 2019 Syed Ibrahim et al., 2019). Consequently, the solar minimum in 2009 was unusually deep, as noted by many authors (e.g., Schrijver et al.,2011). During this period, on several occasions, the solar X-ray flux went below the sensitivity limit of GOES, thereby making accurate flux measurements with GOES not feasible. The SphinX spectrometer with better sensitivity that operated during this time recorded X-ray fluxes that were an order of magnitude below the GOES A1 class (Sylwester et al., 2019).

The minimum between Solar Cycles 24 and 25 in 2019-20 has been deeper than the previous one, and it is expected that the period of low activity may continue longer and may result in a weaker maximum compared to earlier cycles (Bisoi, Janardhan, and Ananthakrishnan, 2020; Bisoi and Janardhan, 2020) . It has been even speculated that the Sun may be headed for an extended period of very low solar activity similar to that experienced in the Maunder minimum (Janardhan et al., 2015b). If this trend continues, there is a need for more sensitive instruments to monitor the solar X-ray flux. As discussed in the previous section, owing to the very low background, the XSM is sensitive enough to measure X-ray fluxes down to a few orders of magnitude below A1 class, and hence would be ideal to monitor the solar X-ray flux during such extremely low levels of solar activity and deep minima. Except for $\approx 40$ days during each of the two 'noon-midnight' seasons every year, observations with the XSM are available every day. Beyond the XSM, data from the X-ray spectrometers (Sankarasubramanian et al., 2017) 
and in-situ particle measurements (Janardhan et al., 2017) on board the AdityaL1 mission will enable one to continue the study of the Sun in this unique low activity phase.

\section{Summary}

The Solar X-ray Monitor on board the Chandrayaan-2 orbiter has been operational in the lunar orbit from September 2019, and it is the only spectrometer currently monitoring the Sun in soft X-rays. Observations of the Sun with the XSM have been continuing as per the plan, and the instrument performance has remained stable. Using the in-flight observations of the onboard calibration source, we have shown that the gain and spectral resolution $(\approx 175 \mathrm{eV})$ are stable over time and they match the ground calibration. The on-ground estimate of the effective area has been refined using in-flight observations, and the uncertainty in the effective area with angle is shown to be within $1 \%$. In-flight observations have also demonstrated that the XSM has the sensitivity to carry out spectral measurements during solar activity well below A class level, which is made possible by the extremely low background.

With these established capabilities, the XSM is expected to contribute to our understanding of the Sun, particularly with the investigations of the sub-A class flares, which are well detected during the current low solar activity period. Although the nominal mission life for Chandrayaan-2 is two years, it is expected that the operations would continue beyond that, which would then provide a unique opportunity to monitor evolution the solar activity during at least the rising phase of the Solar Cycle 25. With the commencement of the nominal operation phase of instruments on board the Solar Orbiter in next year, there will also be opportunities for simultaneous observations with them. Further, these studies can also be continued with the X-ray spectrometers and other instruments of the upcoming Aditya-L1 mission.

Acknowledgments We thank the reviewer for helpful comments and suggestions which helped in improving the presentation of the article. The XSM payload was designed and developed by the Physical Research Laboratory (PRL), Ahmedabad, supported by the Department of Space, Govt. of India. PRL was also responsible for the development of the data processing software, the overall payload operations, and data analysis of the XSM. The filter wheel mechanism for the XSM was provided by the U. R. Rao Satellite Centre (URSC), Bengaluru, along with the Laboratory for Electro-Optics Systems (LEOS), Bengaluru. Thermal design and analysis of the XSM packages were carried out by URSC whereas, the Space Application Centre (SAC) supported in mechanical design and analysis. SAC also supported in the fabrication of the flight model of the payload and its test and evaluation for the flight use. We thank various facilities and the technical teams of all the above centers for their support during the design, fabrication, and testing of this payload. The authors also thank the Chandrayaan-2 mission, operations, and ground segment teams. We thank the CLASS team for useful discussions. The authors acknowledge G. Del-Zanna for his help with the simulations of theoretical spectra using CHIANTI and for useful discussions. The Chandrayaan-2 mission is funded and managed by the Indian Space Research Organisation (ISRO). 
Disclosure of Potential Conflicts of Interest The authors declare that they have no conflicts of interest.

\section{References}

Alha, L., Huovelin, J., Nygård, K., Andersson, H., Esko, E., Howe, C.J., Kellett, B.J., Narendranath, S., Maddison, B.J., Crawford, I.A., Grand e, M., Shreekumar, P.: 2009, Ground calibration of the Chandrayaan-1 X-ray Solar Monitor (XSM). Nuclear Instruments and Methods in Physics Research A 607(3), 544. DOI ADS

Allred, J., Daw, A., Brosius, J.: 2018, A 3D Model of AR 11726 Heated by Nanoflares. arXiv e-prints, arXiv:1807.00763. ADS

Arnaud, K.A.: 1996, XSPEC: The First Ten Years. In: Jacoby, G.H., Barnes, J. (eds.) Astronomical Data Analysis Software and Systems V, Astronomical Society of the Pacific Conference Series 101, 17. ADS

Athiray, P.S., Vievering, J., Glesener, L., Ishikawa, S.-n., Narukage, N., Buitrago-Casas, J.C., Musset, S., Inglis, A., Christe, S., Krucker, S., Ryan, D.: 2020, FOXSI-2 Solar Microflares. I. Multi-instrument Differential Emission Measure Analysis and Thermal Energies. ApJ 891(1), 78. DOI ADS.

Bhardwaj, A., Lisse, C., Dennerl, K.: 2014, X-rays in the Solar System, 1019. DOI.

Bhardwaj, A., Elsner, R.F., Randall Gladstone, G., Cravens, T.E., Lisse, C.M., Dennerl, K., Branduardi-Raymont, G., Wargelin, B.J., Hunter Waite, J., Robertson, I., Østgaard, N., Beiersdorfer, P., Snowden, S.L., Kharchenko, V.: 2007, X-rays from solar system objects. Planet. Space Sci. 55(9), 1135. DOI ADS.

Bisoi, S.K., Janardhan, P.: 2020, A New Tool for Predicting the Solar Cycle: Correlation Between Flux Transport at the Equator and the Poles. Sol. Phys. 295(6), 79. DOI ADS

Bisoi, S.K., Janardhan, P., Ananthakrishnan, S.: 2020, Another Mini Solar Maximum in the Offing: A Prediction for the Amplitude of Solar Cycle 25. Journal of Geophysical Research (Space Physics) 125(7), e27508. DOI ADS.

Brosius, J.W., Davila, J.M., Thomas, R.J., Monsignori-Fossi, B.C.: 1996, Measuring Active and Quiet-Sun Coronal Plasma Properties with Extreme-Ultraviolet Spectra from SERTS. ApJS 106, 143. DOI ADS

Chifor, C., Tripathi, D., Mason, H.E., Dennis, B.R.: 2007, X-ray precursors to flares and filament eruptions. A\&A 472(3), 967. DOI ADS

Cooper, K., Hannah, I.G., Grefenstette, B.W., Glesener, L., Krucker, S., Hudson, H.S., White, S.M., Smith, D.M.: 2020, NuSTAR Observation of a Minuscule Microflare in a Solar Active Region. ApJ 893(2), L40. DOI ADS

Del Zanna, G., Mason, H.E.: 2018, Solar UV and X-ray spectral diagnostics. Living Reviews in Solar Physics 15(1), 5. DOI ADS

Del Zanna, G., Dere, K.P., Young, P.R., Landi, E., Mason, H.E.: 2015, CHIANTI - An atomic database for emission lines. Version 8. A\&A 582, A56. DOI ADS

Dennis, B.R., Phillips, K.J.H., Schwartz, R.A., Tolbert, A.K., Starr, R.D., Nittler, L.R.: 2015, Solar Flare Element Abundances from the Solar Assembly for X-Rays (SAX) on MESSENGER. ApJ 803(2), 67. DOI ADS

Dere, K.P., Landi, E., Mason, H.E., Monsignori Fossi, B.C., Young, P.R.: 1997, CHIANTI an atomic database for emission lines. A\&AS 125, 149. DOI ADS

Dong, W.-D., Zhang, X., Li, Y., Tang, C.-L., Xu, A., Zhang, F.: 2019, The Calibrations for the Chang'E-2 Solar X-Ray Monitor. Sol. Phys. 294(9), 120. DOI ADS

Dudík, J., Dzifč́ková, E., Meyer-Vernet, N., Del Zanna, G., Young, P.R., Giunta, A., Sylwester, B., Sylwester, J., Oka, M., Mason, H.E., Vocks, C., Matteini, L., Krucker, S., Williams, D.R., Mackovjak, S.: 2017, Nonequilibrium Processes in the Solar Corona, Transition Region, Flares, and Solar Wind (Invited Review). Sol. Phys. 292(8), 100. DOI ADS

Fárník, F., Hudson, H., Watanabe, T.: 1996, Spatial Relations between Preflares and Flares. Sol. Phys. 165(1), 169. DOI ADS

Feldman, U., Doschek, G.A., Mariska, J.T., Brown, C.M.: 1995, Relationships between Temperature and Emission Measure in Solar Flares Determined from Highly Ionized Iron Spectra and from Broadband X-Ray Detectors. ApJ 450, 441. DOI ADS

Gburek, S., Sylwester, J., Kowalinski, M., Bakala, J., Kordylewski, Z., Podgorski, P., Plocieniak, S., Siarkowski, M., Sylwester, B., Trzebinski, W., Kuzin, S.V., Pertsov, A.A., 
Kotov, Y.D., Farnik, F., Reale, F., Phillips, K.J.H.: 2013, SphinX: The Solar Photometer in X-Rays. Sol. Phys. 283(2), 631. DOI. ADS

Gupta, G.R., Sarkar, A., Tripathi, D.: 2018, Observation and Modeling of Chromospheric Evaporation in a Coronal Loop Related to Active Region Transient Brightening. ApJ $\mathbf{8 5 7}(2), 137$. DOI ADS

Harrison, F.A., Craig, W.W., Christensen, F.E., Hailey, C.J., Zhang, W.W., Boggs, S.E., Stern, D., Cook, W.R., Forster, K., Giommi, P., Grefenstette, B.W., Kim, Y., Kitaguchi, T., Koglin, J.E., Madsen, K.K., Mao, P.H., Miyasaka, H., Mori, K., Perri, M., Pivovaroff, M.J., Puccetti, S., Rana, V.R., Westergaard, N.J., Willis, J., Zoglauer, A., An, H., Bachetti, M., Barrière, N.M., Bellm, E.C., Bhalerao, V., Brejnholt, N.F., Fuerst, F., Liebe, C.C., Markwardt, C.B., Nynka, M., Vogel, J.K., Walton, D.J., Wik, D.R., Alexander, D.M., Cominsky, L.R., Hornschemeier, A.E., Hornstrup, A., Kaspi, V.M., Madejski, G.M., Matt, G., Molendi, S., Smith, D.M., Tomsick, J.A., Ajello, M., Ballantyne, D.R., Baloković, M., Barret, D., Bauer, F.E., Blandford, R.D., Brandt, W.N., Brenneman, L.W., Chiang, J., Chakrabarty, D., Chenevez, J., Comastri, A., Dufour, F., Elvis, M., Fabian, A.C., Farrah, D., Fryer, C.L., Gotthelf, E.V., Grindlay, J.E., Helfand, D.J., Krivonos, R., Meier, D.L., Miller, J.M., Natalucci, L., Ogle, P., Ofek, E.O., Ptak, A., Reynolds, S.P., Rigby, J.R., Tagliaferri, G., Thorsett, S.E., Treister, E., Urry, C.M.: 2013, The Nuclear Spectroscopic Telescope Array (NuSTAR) High-energy X-Ray Mission. ApJ 770(2), 103. DOI ADS

Hernandez-Perez, A., Su, Y., Veronig, A.M., Thalmann, J., Gömöry, P., Joshi, B.: 2019, Preeruption Processes: Heating, Particle Acceleration, and the Formation of a Hot Channel before the 2012 October 20 M9.0 Limb Flare. ApJ 874(2), 122. DOI ADS

Houck, J.C., Denicola, L.A.: 2000, In: Manset, N., Veillet, C., Crabtree, D. (eds.) ISIS: An Interactive Spectral Interpretation System for High Resolution X-Ray Spectroscopy, Astronomical Society of the Pacific Conference Series 216, 591. ADS

Hudson, H.S.: 1991, Solar flares, microflares, nanoflares, and coronal heating. Sol. Phys. 133(2), 357. DOI ADS

Huovelin, J., Alha, L., Andersson, H., Andersson, T., Browning, R., Drummond, D., Foing, B., Grande, M., Hämäläinen, K., Laukkanen, J., Lämsä, V., Muinonen, K., Murray, M., Nenonen, S., Salminen, A., Sipilä, H., Taylor, I., Vilhu, O., Waltham, N., LopezJorkama, M.: 2002, The SMART-1 X-ray solar monitor (XSM): calibrations for D-CIXS and independent coronal science. Planet. Space Sci. 50(14-15), 1345. DOI. ADS.

Huovelin, J., Vainio, R., Andersson, H., Valtonen, E., Alha, L., Mälkki, A., Grande, M., Fraser, G.W., Kato, M., Koskinen, H., Muinonen, K., Näränen, J., Schmidt, W., Syrjäsuo, M., Anttila, M., Vihavainen, T., Kiuru, E., Roos, M., Peltonen, J., Lehti, J., Talvioja, M., Portin, P., Prydderch, M.: 2010, Solar Intensity X-ray and particle Spectrometer (SIXS). Planet. Space Sci. 58(1-2), 96. DOI ADS.

Jain, R., Dave, H., Shah, A.B., Vadher, N.M., Shah, V.M., Ubale, G.P., Manian, K.S.B., Solanki, C.M., Shah, K.J., Kumar, S., Kayasth, S.L., Patel, V.D., Trivedi, J.J., Deshpande, M.R.: 2005, Solar X-ray Spectrometer (Soxs) Mission on Board GSAT2 Indian Spacecraft: The Low-Energy Payload. Sol. Phys. 227(1), 89. DOI ADS

Janardhan, P., Bisoi, S.K., Ananthakrishnan, S., Tokumaru, M., Fujiki, K.: 2011, The prelude to the deep minimum between solar cycles 23 and 24: Interplanetary scintillation signatures in the inner heliosphere. Geophys. Res. Lett. 38(20), L20108. DOI ADS

Janardhan, P., Bisoi, S.K., Ananthakrishnan, S., Tokumaru, M., Fujiki, K., Jose, L., Sridharan, R.: 2015a, A 20 year decline in solar photospheric magnetic fields: Inner-heliospheric signatures and possible implications. Journal of Geophysical Research (Space Physics) 120(7), 5306. DOI ADS.

Janardhan, P., Bisoi, S.K., Ananthakrishnan, S., Sridharan, R., Jose, L.: 2015b, Solar and Interplanetary Signatures of a Maunder-like Grand Solar Minimum around the Corner Implications to Near-Earth Space. Sun and Geosphere 10, 147. ADS

Janardhan, P., Vadawale, S., Bapat, B., Subramanian, K., Chakrabarty, D., Kumar, P., Sarkar, A., Srivastava, N., Thampi, R., Yadav, V., Dhanya, M., Nampoothiri, G., Abhishek, J., Bhardwaj, A., Subhalakshmi, K.: 2017, Probing the heliosphere using in situ payloads onboard aditya-11. Current Science 113, 620. DOI

Janardhan, P., Fujiki, K., Ingale, M., Bisoi, S.K., Rout, D.: 2018, Solar cycle 24: An unusual polar field reversal. A\&A 618, A148. DOI ADS

Joshi, B., Joshi, A.: 2004, The North-South Asymmetry of Soft X-Ray Flare Index During Solar Cycles 21, 22 and 23. Sol. Phys. 219(2), 343. DOI. ADS 
Joshi, B., Veronig, A.M., Lee, J., Bong, S.-C., Tiwari, S.K., Cho, K.-S.: 2011, Pre-flare Activity and Magnetic Reconnection during the Evolutionary Stages of Energy Release in a Solar Eruptive Flare. ApJ 743(2), 195. DOI ADS.

Joshi, B., Kushwaha, U., Cho, K.-S., Veronig, A.M.: 2013, RHESSI and TRACE Observations of Multiple Flare Activity in AR 10656 and Associated Filament Eruption. ApJ 771(1), 1. DOI. ADS

Joshi, B., Bhattacharyya, R., Pandey, K.K., Kushwaha, U., Moon, Y.-J.: 2015, Evolutionary aspects and north-south asymmetry of soft X-ray flare index during solar cycles 21,22 , and 23. A\&A 582, A4. DOI ADS

Joshi, B., Kushwaha, U., Veronig, A.M., Cho, K.-S.: 2016, Pre-flare Coronal Jet and Evolutionary Phases of a Solar Eruptive Prominence Associated with the M1.8 Flare: SDO and RHESSI Observations. ApJ 832(2), 130. DOI ADS.

Krucker, S., Battaglia, M., Cargill, P.J., Fletcher, L., Hudson, H.S., MacKinnon, A.L., Masuda, S., Sui, L., Tomczak, M., Veronig, A.L., Vlahos, L., White, S.M.: 2008, Hard X-ray emission from the solar corona. A\&A Rev. 16, 155. DOI ADS

Krucker, S., Hurford, G. J., Grimm, O., Kögl, S., -P Gröbelbauer, H., Etesi, L., Casadei, D., Csillaghy, A., Benz, A. O., Arnold, N. G., Molendini, F., Orleanski, P., Schori, D., Xiao, H., Kuhar, M., Kobler, S., Iseli, L., Dreier, M., Wiehl, H. J., Kleint, L., et al.: 2020, The spectrometer/telescope for imaging x-rays (stix). A\&A. DOI.

Kushwaha, U., Joshi, B., Cho, K.-S., Veronig, A., Tiwari, S.K., Mathew, S.K.: 2014, Impulsive Energy Release and Non-thermal Emission in a Confined M4.0 Flare Triggered by Rapidly Evolving Magnetic Structures. ApJ 791(1), 23. DOI. ADS.

Laming, J.M.: 2015, The FIP and Inverse FIP Effects in Solar and Stellar Coronae. Living Reviews in Solar Physics 12(1), 2. DOI ADS

Landi, E., Chiuderi Drago, F.: 2008, The Quiet-Sun Differential Emission Measure from Radio and UV Measurements. ApJ 675(2), 1629. DOI ADS.

Lin, R.P., Dennis, B.R., Hurford, G.J., Smith, D.M., Zehnder, A., Harvey, P.R., Curtis, D.W., Pankow, D., Turin, P., Bester, M., Csillaghy, A., Lewis, M., Madden, N., van Beek, H.F., Appleby, M., Raudorf, T., McTiernan, J., Ramaty, R., Schmahl, E., Schwartz, R., Krucker, S., Abiad, R., Quinn, T., Berg, P., Hashii, M., Sterling, R., Jackson, R., Pratt, R., Campbell, R.D., Malone, D., Landis, D., Barrington-Leigh, C.P., Slassi-Sennou, S., Cork, C., Clark, D., Amato, D., Orwig, L., Boyle, R., Banks, I.S., Shirey, K., Tolbert, A.K., Zarro, D., Snow, F., Thomsen, K., Henneck, R., McHedlishvili, A., Ming, P., Fivian, M., Jordan, J., Wanner, R., Crubb, J., Preble, J., Matranga, M., Benz, A., Hudson, H., Canfield, R.C., Holman, G.D., Crannell, C., Kosugi, T., Emslie, A.G., Vilmer, N., Brown, J.C., Johns-Krull, C., Aschwanden, M., Metcalf, T., Conway, A.: 2002, The Reuven Ramaty High-Energy Solar Spectroscopic Imager (RHESSI). Sol. Phys. 210(1), 3. DOI ADS

Mackovjak, Š., Dzifčáková, E., Dudík, J.: 2014, Differential emission measure analysis of active region cores and quiet Sun for the non-Maxwellian $\kappa$-distributions. A\&A 564, A130. DOI ADS

Masterson, R.A., Chodas, M., Bayley, L., Allen, B., Hong, J., Biswas, P., McMenamin, C., Stout, K., Bokhour, E., Bralower, H., Carte, D., Chen, S., Jones, M., Kissel, S., Schmidt, F., Smith, M., Sondecker, G., Lim, L.F., Lauretta, D.S., Grindlay, J.E., Binzel, R.P.: 2018, Regolith X-Ray Imaging Spectrometer (REXIS) Aboard the OSIRIS-REx Asteroid Sample Return Mission. Space Sci. Rev. 214(1), 48. DOI ADS

Mithun, N.P.S., Vadawale, S.V., Shanmugam, M., Patel, A.R., Tiwari, N.K., Adalja, H.L., Goyal, S.K., Ladiya, T., Singh, N., Kumar, S., Tiwari, M.K., Modi, M.H., Mondal, B., Sarkar, A., Joshi, B., Janardhan, P., Bhardwaj, A.: 2020a, Ground Calibration of Solar Xray Monitor On Board the Chandrayaan-2 Orbiter. Experimental Astronomy (submitted), arXiv:2007.07326. ADS

Mithun, N.P.S., Vadawale, S.V., Patel, A.R., Shanmugam, M., Chakrabarty, D., Konar, P., Sarvaiya, T.N., Padia, G.D., Sarkar, A., Kumar, P., Jangid, P., Sarda, A., Shah, M.S., Bhardwaj, A.: 2020b, Data Processing Software for Chandrayaan-2 Solar X-ray Monitor. Astronomy and Computing (submitted), arXiv:2007.11371. ADS

Mitra, P.K., Joshi, B.: 2019, Preflare Processes, Flux Rope Activation, Large-scale Eruption, and Associated X-class Flare from the Active Region NOAA 11875. ApJ 884(1), 46. DOI ADS

Mitra, P.K., Joshi, B., Prasad, A.: 2020, Identification of Pre-flare Processes and Their Possible Role in Driving a Large-scale Flux Rope Eruption with Complex M-class Flare in the Active Region NOAA 12371. Sol. Phys. 295(2), 29. DOI ADS 
Mitra-Kraev, U., Del Zanna, G.: 2019, Solar microflares: a case study on temperatures and the Fe XVIII emission. A\&A 628, A134. DOI ADS.

Moore, C.S., Caspi, A., Woods, T.N., Chamberlin, P.C., Dennis, B.R., Jones, A.R., Mason, J.P., Schwartz, R.A., Tolbert, A.K.: 2018, The Instruments and Capabilities of the Miniature X-Ray Solar Spectrometer (MinXSS) CubeSats. Sol. Phys. 293(2), 21. DOI ADS

Narendranath, S., Sreekumar, P., Alha, L., Sankarasubramanian, K., Huovelin, J., Athiray, P.S.: 2014a, Elemental Abundances in the Solar Corona as Measured by the X-ray Solar Monitor Onboard Chandrayaan-1. Sol. Phys. 289(5), 1585. DOI ADS

Narendranath, S., Tadeppalli, S.P., Athiray, P.S., Misra, A., Sreekumar, P.: 2014b, Observations of the Geotail While in Lunar Orbit by the Chandrayaan-1 X-Ray Spectrometer (C1XS). In: Lunar and Planetary Science Conference, Lunar and Planetary Science Conference, 2199. ADS.

Narendranath, S., Vadodariya, K., Pillai, N.S., Radhakrishna, V., Tyagi, A., Vadawale, S., Mithun, N.P.S., Shanmugam, M., Patel, A., Tadepalli, S.P., Yadav, R., Singh, B., Sharan, V., Sreekumar, P., Bhatt, M., Satya, N., Shetty, A., Suresha Kumar, H.N., Balaji, G., Kumar, K., Raghavendra, V., Agarwal, A.: 2020, Lunar Elemental Abundances from Chandrayaan-2 Large Area Soft X-Ray Spectrometer (CLASS). In: Lunar and Planetary Science Conference, Lunar and Planetary Science Conference, 1186. ADS

Prigozhin, G., Gendreau, K., Doty, J.P., Foster, R., Remillard, R., Malonis, A., LaMarr, B., Vezie, M., Egan, M., Villasenor, J., Arzoumanian, Z., Baumgartner, W., Scholze, F., Laubis, C., Krumrey, M., Huber, A.: 2016, NICER instrument detector subsystem: description and performance. In: Space Telescopes and Instrumentation 2016: Ultraviolet to Gamma Ray, Society of Photo-Optical Instrumentation Engineers (SPIE) Conference Series 9905, 99051I. DOI ADS

Radhakrishna, V., Tyagi, A., Narendranath, S., Vadodariya, K., Yadav, R., Singh, B., Balaji, G., Satya, N., Shetty, A., Suresh Kumar, H.N., Kumar, Vaishali, S., Pillai, N.S., Tadepalli, S., Raghavendra, V., Sreekumar, P., Agarwal, A., Valarmathi, N.: 2020, Chandrayaan-2 Large Area Soft X-ray Spectrometer. Current Science 118(2), 219. DOI

Reale, F., Testa, P., Klimchuk, J.A., Parenti, S.: 2009, Evidence of Widespread Hot Plasma in a Nonflaring Coronal Active Region from Hinode/X-Ray Telescope. ApJ 698(1), 756. DOI] ADS

Sahu, S., Joshi, B., Mitra, P.K., Veronig, A.M., Yurchyshyn, V.: 2020, Hard X-Ray Emission from an Activated Flux Rope and Subsequent Evolution of an Eruptive Long-duration Solar Flare. ApJ 897(2), 157. DOI ADS

Saint-Hilaire, P., Benz, A.O.: 2005, Thermal and non-thermal energies of solar flares. A\&A 435(2), 743. DOI ADS

Sankarasubramanian, K., Sudhakar, M., Nandi, A., m c, R., Adoni, A.A., Kushwaha, A., Agarwal, A., Dey, A., Joshi, B., Singh, B., Girish, V., Tomar, I., Majhi, K.K., Kumar, K., Olekar, M., Bug, M., Pala, M., Thakur, M.K., Badagandi, R., Yougandar, B.: 2017, X-ray spectrometers on-board aditya-11 for solar flare studies. Current Science 113, 625. DOI

Sasikumar Raja, K., Janardhan, P., Bisoi, S.K., Ingale, M., Subramanian, P., Fujiki, K., Maksimovic, M.: 2019, Global Solar Magnetic Field and Interplanetary Scintillations During the Past Four Solar Cycles. Sol. Phys. 294(9), 123. DOI. ADS.

Schlemm, C.E., Starr, R.D., Ho, G.C., Bechtold, K.E., Hamilton, S.A., Boldt, J.D., Boynton, W.V., Bradley, W., Fraeman, M.E., Gold, R.E., Goldsten, J.O., Hayes, J.R., Jaskulek, S.E., Rossano, E., Rumpf, R.A., Schaefer, E.D., Strohbehn, K., Shelton, R.G., Thompson, R.E., Trombka, J.I., Williams, B.D.: 2007, The X-Ray Spectrometer on the MESSENGER Spacecraft. Space Sci. Rev. 131(1-4), 393. DOI ADS

Schonfeld, S.J., White, S.M., Hock-Mysliwiec, R.A., McAteer, R.T.J.: 2017, The Slowly Varying Corona. I. Daily Differential Emission Measure Distributions Derived from EVE Spectra. ApJ 844(2), 163. DOI. ADS.

Schrijver, C.J., Livingston, W.C., Woods, T.N., Mewaldt, R.A.: 2011, The minimal solar activity in 2008-2009 and its implications for long-term climate modeling. Geophys. Res. Lett. 38(6), L06701. DOI ADS

Shanmugam, M., Vadawale, S.V., Patel, A.R., Adalaja, H.K., Mithun, N.P.S., Ladiya, T., Goyal, S.K., Tiwari, N.K., Singh, N., Kumar, S., Painkra, D.K., Acharya, Y.B., Bhardwaj, A., Hait, A.K., Patinge, A., Kapoor, A.h., Kumar, H.N.S., Satya, N., Saxena, G., Arvind, K.: 2020, Solar X-ray Monitor Onboard Chandrayaan-2 Orbiter. Current Science 118(1), 45. DOI

Shimizu, T.: 1995, Energetics and Occurrence Rate of Active-Region Transient Brightenings and Implications for the Heating of the Active-Region Corona. PASJ 47, 251. ADS 
Syed Ibrahim, M., Joshi, B., Cho, K.-S., Kim, R.-S., Moon, Y.-J.: 2019, Interplanetary Coronal Mass Ejections During Solar Cycles 23 and 24: Sun-Earth Propagation Characteristics and Consequences at the Near-Earth Region. Sol. Phys. 294(5), 54. DOI ADS

Sylwester, B., Sylwester, J., Phillips, K.J.H., Kepa, A., Mrozek, T.: 2014, Solar Flare Composition and Thermodynamics from RESIK X-Ray Spectra. ApJ 787(2), 122. DOI ADS

Sylwester, B., Phillips, K.J.H., Sylwester, J., Kepa, A.: 2015, Resik Solar X-Ray Flare Element Abundances on a Non-isothermal Assumption. ApJ 805(1), 49. DOI ADS

Sylwester, B., Sylwester, J., Siarkowski, M., Phillips, K.J.H., Podgorski, P., Gryciuk, M.: 2019, Analysis of Quiescent Corona X-ray Spectra from SphinX During the 2009 Solar Minimum. Sol. Phys. 294(12), 176. DOI. ADS

Trombka, J.I., Nittler, L.R., Starr, R.D., Evans, L.G., McCoy, T.J., Boynton, W.V., Burbine, T.H., Brückner, J., Gorenstein, P., Squyres, S.W., Reedy, R.C., Goldsten, J.O., Lim, L., Hurley, K., Clark, P.E., Floyd, S.R., McClanahan, T.P., McCartney, E., Branscomb, J., Bhangoo, J.S., Mikheeva, I., Murphy, M.E.: 2001, The NEAR-Shoemaker x-ray/gamma-ray spectrometer experiment: Overview and lessons learned. Meteoritics and Planetary Science 36(12), 1605. DOI ADS

Türler, M., Chernyakova, M., Courvoisier, T.J.-L., Lubiński, P., Neronov, A., Produit, N., Walter, R.: 2010, INTEGRAL hard X-ray spectra of the cosmic X-ray background and Galactic ridge emission. A\&A 512, A49. DOI ADS.

Vanitha, M., Veeramuthuvel, P., Kalpana, K., Nagesh, G.: 2020, Chandrayaan-2: The Second Indian Mission to the Moon. In: Lunar and Planetary Science Conference, Lunar and Planetary Science Conference, 1994. ADS

Wright, P.J., Hannah, I.G., Grefenstette, B.W., Glesener, L., Krucker, S., Hudson, H.S., Smith, D.M., Marsh, A.J., White, S.M., Kuhar, M.: 2017, Microflare Heating of a Solar Active Region Observed with NuSTAR, Hinode/XRT, and SDO/AIA. ApJ 844(2), 132. DOI ADS 